\title{
A Remorin from Nicotiana benthamiana Interacts with the Pseudomonas Type-III Effector Protein HopZ1a and is Phosphorylated by the Immune-Related Kinase PBS1
}

\author{
Philip Albers, ${ }^{1}$ Suayib Üstün, ${ }^{1}$ Katja Witzel, ${ }^{2}$ Max Kraner, ${ }^{3}$ and Frederik Börnke ${ }^{1,4, \dagger}$ \\ ${ }^{1}$ Plant Metabolism, Leibniz-Institute of Vegetable and Ornamental Crops (IGZ), 14979 Großbeeren, Germany \\ ${ }^{2}$ Principles of Integrated Pest Management, Leibniz-Institute of Vegetable and Ornamental Crops (IGZ), 14979 Großbeeren, \\ Germany \\ ${ }^{3}$ Friedrich-Alexander-Universität, Department of Biology, Division of Biochemistry, 91058 Erlangen, Germany \\ ${ }^{4}$ Institute of Biochemistry and Biology, University of Potsdam, 14476 Potsdam, Germany
}

Accepted 18 April 2019.

\begin{abstract}
The plasma membrane $(P M)$ is at the interface of plantpathogen interactions and, thus, many bacterial type-III effector (T3E) proteins target membrane-associated processes to interfere with immunity. The Pseudomonas syringae T3E HopZ1a is a host cell PM-localized effector protein that has several immunity-associated host targets but also activates effector-triggered immunity in resistant backgrounds. Although HopZ1a has been shown to interfere with early defense signaling at the PM, no dedicated PM-associated HopZ1a target protein has been identified until now. Here, we show that HopZ1a interacts with the PM-associated remorin protein NbREM4 from Nicotiana benthamiana in several independent assays. NbREM4 relocalizes to membrane nanodomains after treatment with the bacterial elicitor flg22 and transient overexpression of NbREM4 in $N$. benthamiana induces the expression of a subset of defenserelated genes. We can further show that NbREM4 interacts with the immune-related receptor-like cytoplasmic kinase avrPphBsusceptible 1 (PBS1) and is phosphorylated by PBS1 on several residues in vitro. Thus, we conclude that NbREM4 is associated with early defense signaling at the PM. The possible relevance of the HopZ1a-NbREM4 interaction for HopZ1a virulence and avirulence functions is discussed.
\end{abstract}

Keywords: bacterial pathogenesis, defense signaling pathways, effectors, elicitors, HopZ1a, MAMPs, PAMPs, PBS1, Pseudomonas syringae, remorin, type-3 secretion

Plants possess a sophisticated and multilayered immune system that generally prevents infection by potential pathogens

Current address for S. Üstün: Centre for Plant Molecular Biology, Molecular Genetics, University of Tübingen, 72076 Tübingen, Germany.

${ }^{\dagger}$ Corresponding author: F. Börnke; boernke@igzev.de

Funding: This work was supported by grants from the Deutsche Forschungsgemeinschaft (BO1916/5-1 and BO1916/5-2) to F. Börnke.

*The $e$-Xtra logo stands for "electronic extra" and indicates that 11 supplementary figures and 1 supplementary table are published online.

The author(s) declare no conflict of interest.

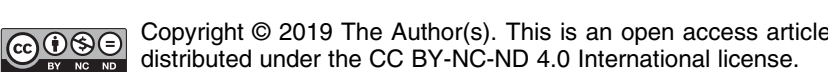

(Dodds and Rathjen 2010; Jones and Dangl 2006). This type of resistance is usually based on the recognition of conserved microbe-associated molecular patterns (MAMPs) by plant cellsurface-localized pattern recognition receptors (PRRs) which, upon activation, trigger a suite of defense responses collectively preventing ingress of invading pathogens and leading to socalled pattern-triggered immunity (PTI) (Macho and Zipfel 2015). However, adapted pathogens have evolved virulence strategies to overcome plant defense and to cause disease in a given host species. The injection of type III effector (T3E) proteins into the host cell is an efficient mechanism employed by many Gram-negative bacterial pathogens to suppress plant immunity and promote disease development (Büttner 2016; Khan et al. 2018a). T3Es encode proteins with diverse biochemical activities in order to interfere with host cellular processes, including proteases, acetyltransferases, E3-ubiquitin ligases, phosphatases, and protein kinases, to name just a few. Host targets of T3Es include signaling proteins, transcriptional regulators, the protein processing machinery, as well as metabolic enzymes, with a majority of targets involved in plant immunity (Büttner 2016; Khan et al. 2018a). Typically, 20 to 40 T3Es are translocated from a given bacterium into the host cell that collectively dampen the PTI response below a threshold allowing for bacterial multiplication and disease progression during so-called effector-triggered susceptibility (Jones and Dangl 2006).

As a response, plants have evolved the ability to either directly or indirectly recognize specific effector proteins through resistance $(\mathrm{R})$ proteins, a class of intracellular receptor proteins that typically contain nucleotide-binding domains (NB) and leucine-rich repeats (LRRs). Effector recognition by NB-LRR proteins results in an accelerated and amplified PTI response and, in most cases, leads to localized cell death at the site of infection termed hypersensitive response (HR), eventually inducing effector-triggered immunity (ETI) (Cesari 2018; Dodds and Rathjen 2010).

The T3E HopZ1a from Pseudomonas syringae pv. syringae A2 strain is a member of the widely distributed YopJ superfamily of cysteine proteases and acetyltransferases produced by both plant and animal bacterial pathogens (Lewis et al. 2011). It is a low-specificity $\mathrm{T} 3 \mathrm{E}$ that has several distinct molecular targets in different plant species (Khan et al. 2018a). HopZ1a possesses acetyltransferase activity and has been shown to acetylate tubulin, which disrupts the plant cytoskeletal network, resulting in breakdown of cellular trafficking (Lee 
et al. 2012). In soybean, HopZ1a has been demonstrated to interact with the isoflavone biosynthesis enzyme 2-hydroxyisoflavanone dehydratase (GmHID1). Binding of HopZ1a promotes GmHID1 degradation and subsequently increases susceptibility of soybean to $P$. syringae by decreasing the capacity of the host to synthesize the phytoalexin daidzein (Zhou et al. 2011). Other host targets of HopZ1a include jasmonate ZIM-domain (JAZ) proteins, which negatively regulate the expression of jasmonic acid (JA)-responsive genes. Acetylation of JAZ proteins by HopZ1a mediates their degradation and, thus, activates JA signaling (Jiang et al. 2013). Activation of JA signaling antagonizes salicylic acid (SA)mediated defense responses, which are required for immunity against hemibiotrophic pathogens such $P$. syringae (Zheng et al. 2012).

Transgenic expression of HopZ1a in Arabidopsis has been shown to suppress several outputs of PTI such as the production of reactive oxygen species (ROS) and the activation of mitogen-activated protein (MAP) kinase signaling (Lewis et al. 2014). These effects are not well explained by the current known HopZ1a target proteins and thus additional targets might exist.

HopZ1a has been shown to trigger an HR in Arabidopsis thaliana accession Columbia-0 (Col-0), rice, certain soybean genotypes, and Nicotiana benthamiana (Ma et al. 2006). In Arabidopsis, HopZ1a recognition depends on the NB-LRR protein HopZ-activated resistance 1 (ZAR1) and the HopZETI-deficient 1 (ZED1) pseudokinase (Lewis et al. 2010, 2013). ZED1 itself does not possess kinase activity and the current model suggests that it interacts with ZAR1 and HopZ1a and is acetylated by HopZ1a, which is hypothesized to trigger the activation of ZAR1 (Lewis et al. 2013). Thus, ZED1 appears to be a decoy guarded by ZAR1 and senses the activity of HopZ1a in the plant cell. Recent evidence suggests that, in Arabidopsis, ZAR1 also recognizes the Xanthomonas campestris T3E AvrAC, requiring the ZED1-related kinase 1 (ZRK1) instead of ZED1, as well as the $P$. syringae T3E HopF2a in a ZRK3-dependent manner (Seto et al. 2017; Wang

pBD-P53 / pAD-SV40

pBD-HopZ1a / pAD-NtRemorin

pBD / pAD-NtRemorin

pBD-HopZ1a / pAD-NbRemorin

pBD / pAD-NbRemorin

pBD-HopZ1a / pAD

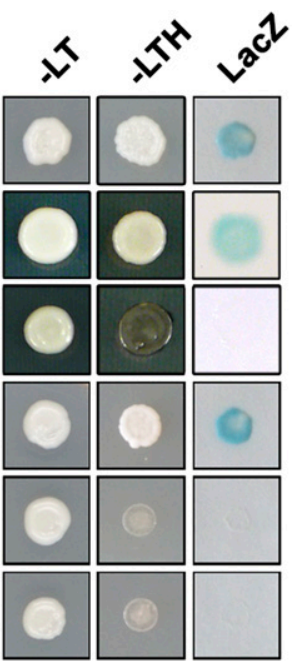

Fig. 1. HopZ1a interacts with a remorin in yeast two-hybrid assays. HopZ1a fused to the GAL4 DNA binding domain (BD) was expressed in combination with remorin fused to the GAL4 activation domain (AD) in yeast strain Y190. Cells were grown on selective media before a LacZ filter assay was performed. pSV40/p53 served as positive control while the empty AD vector served as negative control. NtRemorin = Nicotiana tabacum remorin; NbRemorin $=N$. benthamiana remorin; $-\mathrm{LT}=$ yeast growth on medium without Leu and Trp; -HLT = yeast growth on medium lacking His, Leu, and Trp, indicating expression of the HIS3 reporter gene; and $\mathrm{LacZ}=$ activity of the $l a c Z$ reporter gene. et al. 2015). A ZAR1 ortholog was recently identified in $N$. benthamiana (Baudin et al. 2017); however, opposite to earlier observations (Ma et al. 2006), HopZ1a only triggered a ZAR1-dependent HR in $N$. benthamiana upon coexpression with Arabidopsis ZED1 (Baudin et al. 2017). Thus, it is currently unclear what the endogenous guardee of ZAR1 in $N$. benthamiana is and whether this is a decoy to lure HopZ1a into the ZAR1 immune complex. NbZAR1 was recently shown to be also responsible for the perception of the $X$. perforans T3E XopJ4 in $N$. benthamiana (Schultink et al. 2019). Additional data suggest that XopJ4 perception by NbZAR1 was mediated by the pseudokinase JIM2, a member of the same gene family as ZED1, indicating a mode of action similar to ZAR1 in N. benthamiana as compared with Arabidopsis and suggesting that ZAR1 is involved in the perception of different effector proteins with specificity directed by associated kinase or pseudokinase proteins (Schultink et al. 2019).

In this study, we identified a remorin protein (NbREM4) as a novel interaction partner of HopZ1a in N. benthamiana, and further studies suggest that NbREM4 interacts with the immune-related receptor-like cytoplasmic kinase (RLCK) avrPphB-susceptible 1 (PBS1). Biochemical studies suggest that PBS1 can specifically phosphorylate NbREM4 in vitro on several residues. A possible role of the PBS1-NbREM4 interaction module as a target for HopZ1a is discussed. We further show that HopZ1a triggers a ZAR1-dependent HR in $N$. benthamiana, suggesting that an HopZ1a targets a thus-farunknown guardee of ZAR1 in this plant species.

\section{RESULTS}

\section{HopZ1a interacts with a remorin from $N$. benthamiana in yeast.}

HopZ1a is known to have multiple target proteins in different plant species (Jiang et al. 2013; Lee et al. 2012; Zhou et al. 2011). However, the target proteins known to date do not explain all HopZ1a-related phenotypes observed upon expression of the effector in plants (Lewis et al. 2014). Thus, HopZ1a is likely to possess additional, as-yet-unidentified target proteins. In order to further understand HopZ1a function, we screened for proteins that interact with HopZ1a using a yeast two-hybrid (Y2H) cDNA library from tobacco (N. tabacum). This repeatedly identified different clones of a cDNA encoding a protein with high similarity to remorin-like proteins from different plant species (GenBank accession number XP_016479648.1), which we tentatively named NtRemorin. Remorins are plant-specific plasma membrane (PM) proteins, which may act as molecular scaffolds regulating signal transduction (Jarsch and Ott 2011). One clone isolated during the screening that comprised the entire predicted NtRemorin open reading frame, except the start methionine, was used in a direct $\mathrm{Y} 2 \mathrm{H}$ assay with HopZ1a. Reporter gene activation confirms the interaction between the two proteins in yeast (Fig. 1). We sought to use $N$. benthamiana for further functional analysis of the HopZ1a-remorin interaction because of its amenability to molecular and cell biology manipulations (Goodin et al. 2008). A homology search identified a remorinlike protein with high similarity $(90 \%)$ to NtRemorin encoded by the $N$. benthamiana genome (Niben101Scf00735g05005.1), tentatively dubbed NbRemorin (Supplementary Fig. S1). A direct $\mathrm{Y} 2 \mathrm{H}$ interaction assay suggests that NbRemorin also binds to HopZ1a in yeast and, thus, is suitable for a functional analysis of the interaction (Fig. 1). In Arabidopsis, the remorin family consists of 16 members which fall into six different groups (Raffaele et al. 2007), whereas $N$. benthamiana has recently been shown to harbor at least 35 candidate remorin proteins falling into five groups (Fu et al. 2018). When grouped into a combined phylogenetic tree of the Arabidopsis and $N$. benthamiana 
remorin protein family members, NbRemorin most closely associates with group 4 remorins (Supplementary Fig. S2). A1though Arabidopsis possesses only two group 4 remorins, $N$. benthamiana harbors at least four members of this group which appear to be more closely related to each other than to any of the two Arabidopsis REM4 proteins. Thus, we will refer to NbRemorin as NbREM4 from now on, without making any further subdistinction within this group yet. In order to investigate whether HopZ1a can also interact with other members of the $N$. benthamiana remorin family, two additional remorin isoforms, one from group 1 (NbRem1) and one from group 6 (NbRem6), were tested for their ability to bind HopZ1a in yeast. A direct $\mathrm{Y} 2 \mathrm{H}$ assay suggests no interaction of HopZ1a with NbRem1 or NbRem6 (Supplementary Fig. S3). Thus, although binding of HopZ1a to other remorins not tested in this experiment cannot be excluded, there appears to be at least some specificity of HopZ1a to interact with NbREM4.

The NbREM4 polypeptide comprises 296 amino acids and has a predicted molecular weight of $33 \mathrm{kDa}$. It features the typical domain structure found in remorins from different plant species (Raffaele et al. 2007), with a conserved C-terminal signature region (Pfam domain Remorin_C; PF03763) that encodes a predicted coiled-coil (CC) motif, a putative membraneanchoring motif, and a variable N-terminal part. In order to map the region of HopZ1a binding to NbREM4, we cotransformed constructs comprising the NbREM4 N-terminal region or the $\mathrm{C}$-terminal region of the protein together with HopZ1a in a yeast reporter strain. The conserved NbREM $4 \mathrm{C}$ terminus is necessary and sufficient for the interaction with HopZ1a (Fig. 2). However, the apparently weaker interaction indicates that the N-terminal part of the protein likely also contributes to HopZ1a binding.

A

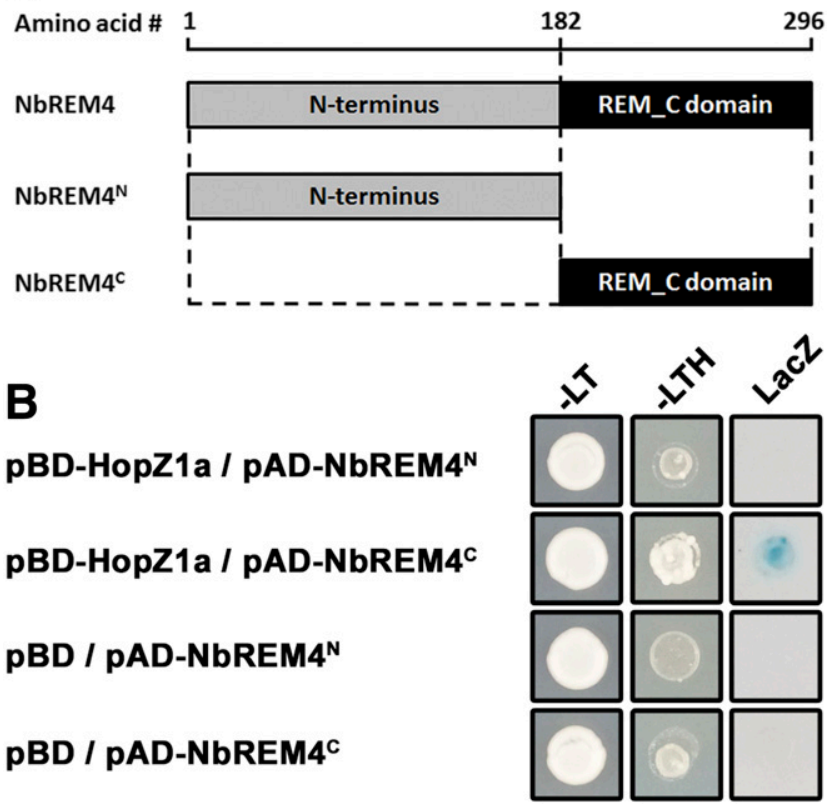

Fig. 2. The NbREM4 $\mathrm{C}$ terminus is required and sufficient for HopZ1a interaction in yeast. A, Schematic representation of the NbREM4 domain structure. B, HopZ1a interacts with the NbREM4 C-terminal region (NbREM4C) comprising amino acids 182 to 296 but not with the $\mathrm{N}$ terminus of NbREM4 (NbREMN) covering amino acids 1 to 181. Cells were grown on selective media before a LacZ filter assay was performed. pSV40/p53 served as positive control while the empty binding domain vector served as negative control. $-\mathrm{LT}=$ yeast growth on medium without Leu and Trp; -HLT = yeast growth on medium lacking His, Leu, and Trp, indicating expression of the HIS3 reporter gene; and LacZ = activity of the lacZ reporter gene.
Remorins have been reported to form hetero- and homooligomers; therefore, we tested the ability of NbREM4 to selfinteract or to bind to other remorin isoforms. A direct $\mathrm{Y} 2 \mathrm{H}$ assay indicates that, indeed, NbREM4 is able to form homomers in yeast but does not bind to two other remorin isoforms tested (Supplementary Fig. S4). To confirm this interaction in planta, we performed bimolecular fluorescence complementation (BiFC) using $N$. benthamiana leaves, where a robust fluorescence signal indicated oligomerization of NbREM4 at the PM of infiltrated tobacco epidermal cells.

HopZ1a interacts with NbREM4 in planta and in vitro.

Next, the subcellular localization of both protein partners in planta was examined to determine whether colocalization as a prerequisite of in vivo interaction can be observed. The green fluorescent protein (GFP) was fused to the $\mathrm{C}$ terminus of HopZ1 ${ }^{\mathrm{C} / \mathrm{A}}$ carrying a cysteine to alanine substitution at position C216 within the conserved catalytic triad of the effector, and the fusion protein was expressed in leaves of $N$. benthamiana using Agrobacterium infiltration. The HopZ1a ${ }^{\mathrm{C} / \mathrm{A}}$ variant was used for the experiments to prevent elicitation of an HR in $N$. benthamiana that has been shown to depend on its catalytic activity (Ma et al. 2006). In accordance with previous findings and the presence of a myristoylation motif at glycine 2 of the HopZ1a (Lewis et al. 2008), HopZ1a ${ }^{\text {C/A }}$-GFP expression generated a fluorescence signal in the periphery of the cell, indicating PM localization of the fusion protein (Fig. 3A). In a similar approach, NbREM4 was tagged at the $\mathrm{N}$ terminus with GFP and transient expression of the GFP-NtRem4 protein in $N$. benthamiana yielded a fluorescence signal resembling that of a PM-associated protein (Fig. 3B). To further corroborate this finding, membranes of GFP-NbREM4 expressing cells were labeled with the fluorescence dye FM4-64 and microscopic analysis revealed substantial overlap between the GFP and the FM4-64 fluorescence signal, confirming PM localization of NbREM4 (Fig. 3B). Thus, both HopZ1a and NbREM4 localize to the plant cell PM and, therefore, could interact in vivo.

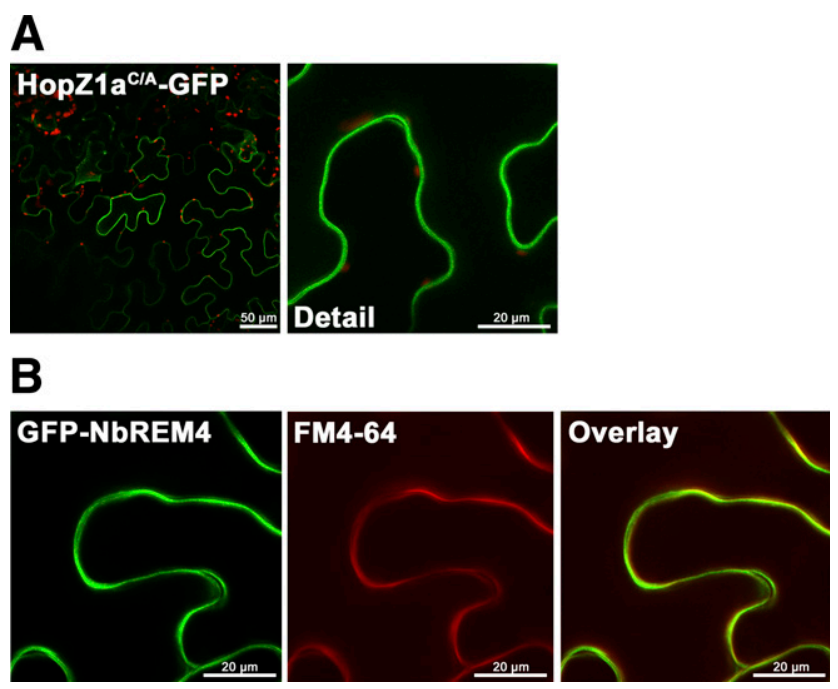

Fig. 3. Subcellular localization of HopZ1a and NbREM4 in planta. A, Subcellular localization of HopZ1a ${ }^{\mathrm{C} / \mathrm{A}}$-green fluorescent protein (GFP) in Nicotiana benthamiana leaves transiently transformed by Agrobacterium infiltration. For confocal laser-scanning microscopy, samples were taken 48 $\mathrm{h}$ postinfiltration (hpi). B, Subcellular localization of GFP-NbREM4. Membranes were stained with FM4-64 (middle) and green and red fluorescence channels were recorded separately to prevent bleed through. The resulting fluorescence images were merged (right). Pictures were taken 48 hpi. 
To verify the interaction of HopZ1a and NbREM4 in planta, BiFC assays were performed in $N$. benthamiana using transient expression via agrobacteria. Strong yellow fluorescent protein (YFP) fluorescence was observed when a combination of HopZ1a-Venus ${ }^{\mathrm{C}}$ with Venus ${ }^{\mathrm{N}}$-NbREM4 was expressed, demonstrating that both proteins interact inside plant cells (Fig. 4A). In accordance with PM localization of HopZ1a as well as of NbREM4, the YFP signal appeared to be confined to the PM because no fluorescence surrounding the chloroplasts could be detected, which would be indicative of a cytosolic localization
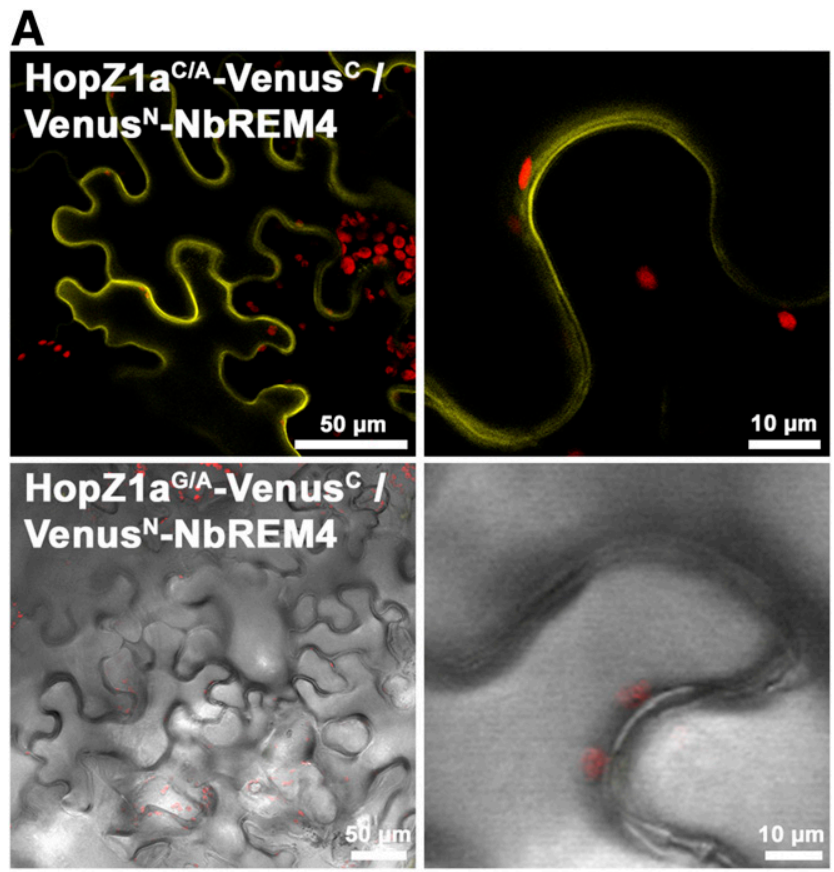

B

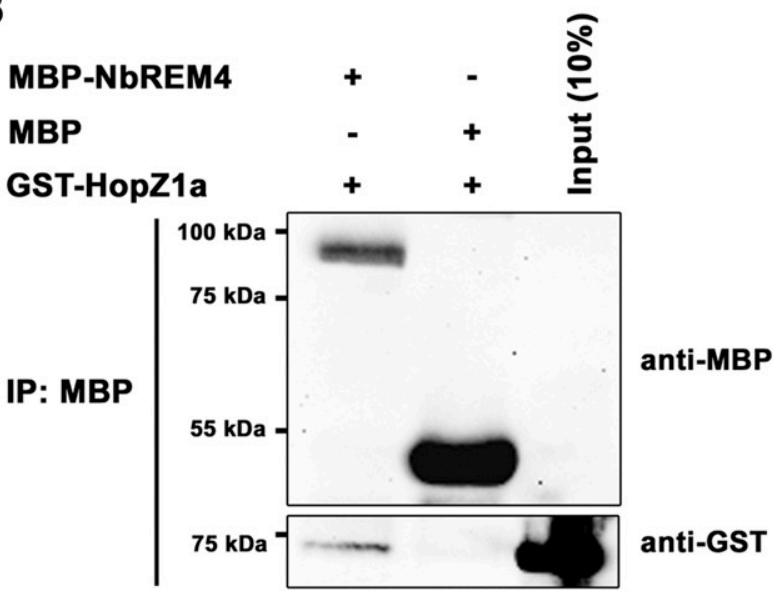

Fig. 4. Interaction of HopZ1a with NbREM4 in planta and in vitro. A, Bimolecular fluorescence complementation in planta interaction studies of HopZ1a variants with NbREM4. Yellow fluorescent protein (YFP) confocal microscopy images show Nicotiana benthamiana leaf epidermal cells transiently expressing HopZ1 $\mathrm{a}^{\mathrm{C} / \mathrm{A}}-$ Venus $^{\mathrm{C}}$ in combination with Venus ${ }^{\mathrm{N}}$. NbREM4. A close-up of the same cells shows that the YFP fluorescence of HopZ1a ${ }^{\mathrm{C} / \mathrm{A}}$-Venus ${ }^{\mathrm{C}} /$ Venus $^{\mathrm{N}}$-NbREM4 aligns with the plasma membrane. The lower panel shows an overlay of fluorescence and differential interference contrast images of leave epidermal cells expressing a combination of Venus $^{\mathrm{N}}$-NbREM4 and HopZ1a ${ }^{\mathrm{G} / \mathrm{A}}$-Venus ${ }^{\mathrm{C}}$, indicating that a mutation at the myristoylation site at G2 of HopZ1a abolishes the interaction with NbREM4. B, In vitro pull-down assay showing physical interaction of HopZ1a with NbREM4. Maltose-binding protein (MBP)NbREM4 and glutathione S-transferase (GST)-HopZ1a were expressed in Escherichia coli. Pull down was performed using amylose resin. Proteins were detected in an immunoblot using antibodies, as indicated. of the interaction (Fig. 4A). Negative controls, including unrelated proteins, yielded no fluorescence signal, indicating the specificity of the interaction (Supplementary Fig. S5). In addition, mutation of the myristoylation motif at glycine 2 of

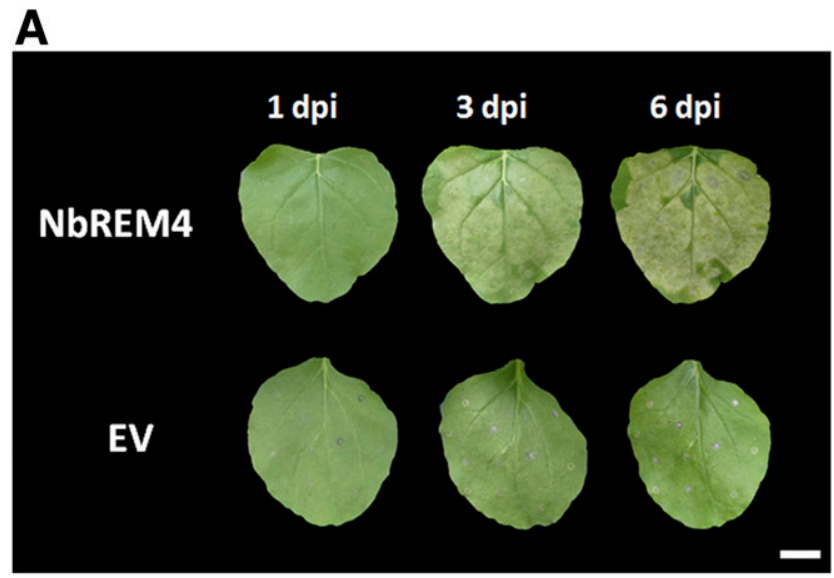

B

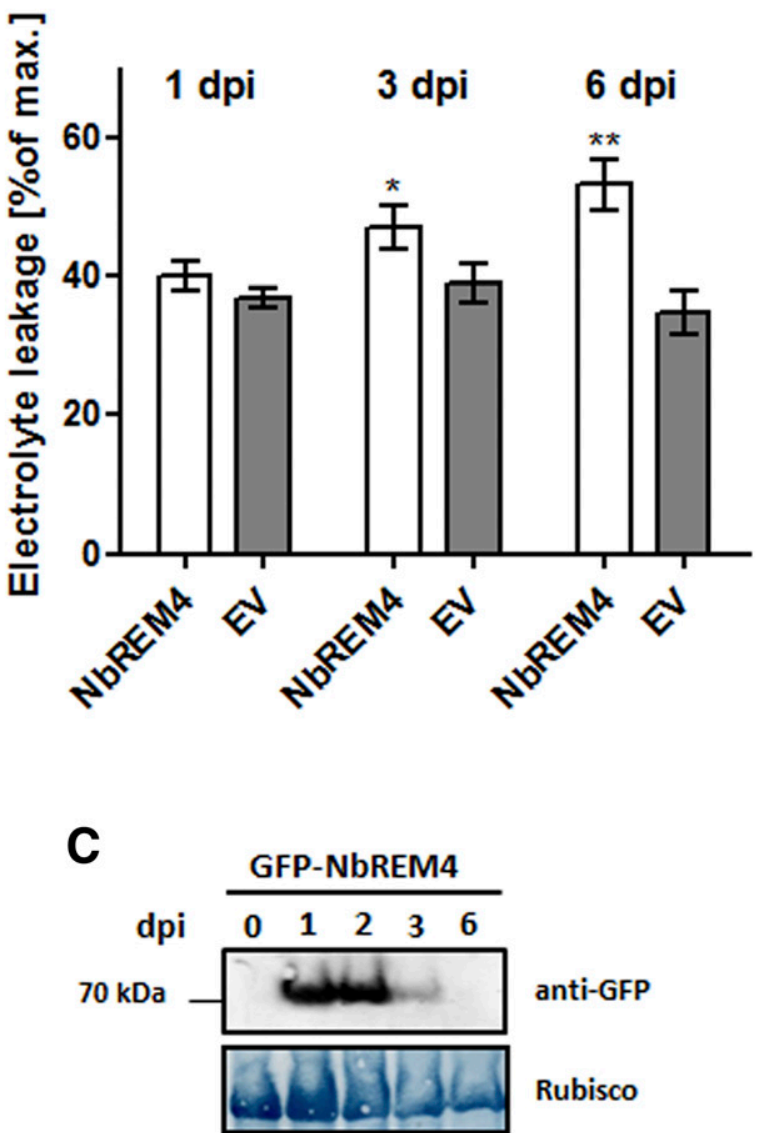

Fig. 5. Transient overexpression of NbREM4 in leaves of Nicotiana ben thamiana leads to tissue damage. A, Time course of phenotype development in $N$. benthamiana leaves infiltrated with Agrobacterium tumefaciens strains that mediate T-DNA-based transfer of NbREM4 and empty vector (EV); dpi $=$ days postinfiltration. $\mathbf{B}$, Ion leakage was measured in plants transiently expressing NbREM4 and EV at time points indicated. Bars represent the average ion leakage measured for triplicates of six leaf disks each, and the error bars indicate standard deviation. Asterisks indicate a significant difference (* and ** indicate $P<0.05$ and 0.01 , respectively) based on results of a Student's $t$ test. The experiment has been repeated three times with similar results. C, Protein extracts from $N$. benthamiana leaves transiently expressing green fluorescent protein (GFP)-NbREM4 at the time points indicated were prepared. Equal volumes representing approximately equal protein amounts of each extract were immunoblotted and proteins were detected using antiGFP antiserum. Amido-black staining served as a loading control. 
HopZ1a abolished the interaction with NbREM4 (Fig. 4A) which is in accordance with a release of the HopZ1 ${ }^{\mathrm{G} / \mathrm{A}}$ protein from the PM (Lewis et al. 2008). Proper expression of all proteins in the BiFC assays has been verified by Western blotting.

To exclude the possibility that the interaction between HopZ1a and NbREM4 is mediated by a third eukaryotic protein, an in vitro pull-down assay was performed. To this end, recombinant glutathione S-transferase (GST)-tagged HopZ1a was incubated with maltose-binding protein (MBP)-tagged NbREM4. Protein complexes were pulled down using amylose resin, which binds MBP, and precipitated proteins were subsequently detected using either anti-GST or anti-MBP antibodies. A Western blot revealed that GST-HopZ1a was pulled down together with MBP-NbREM4, demonstrating a direct physical interaction of both proteins which does not require additional factors (Fig. 4B). MBP alone was not able to pull down GST-HopZ1a, indicating specificity of the in vitro interaction (Fig. 4B).

Taken together, these data suggest that the Pseudomonas T3E HopZ1a directly interacts with the remorin NbREM4 at the PM of plant cells.

\section{Overexpression of NbREM4 affects defense gene expression.}

Remorins from different plant species have been associated with plant defense responses (Bozkurt et al. 2014; Fu et al. 2018; Jarsch and Ott 2011; Son et al. 2014). Given the fact that the majority of Pseudomonas T3Es target immunity-related functions inside the host cell (Büttner 2016; Khan et al. 2018a), the interaction of NbREM4 with HopZ1a points toward an involvement of NbREM4 in some sort of plant defense response. In order to provide first insights into an in planta function of NbREM4, a GFP-tagged version of the protein under the control or the constitutive CaMV35S promoter was transiently expressed in leaves of $N$. benthamiana using Agrobacterium infiltration. A phenotypic inspection of GFPNbREM4-expressing leaves revealed that overexpression of the remorin protein led to the development of chloroses in infiltrated areas 3 days postinfiltration (dpi), which progressed into tissue collapse until 6 dpi (Fig. 5A). An anti-GFP Western blot suggests that GFP-NbREM4 protein levels are highest 1 and 2 dpi and decline to almost undetectable levels until 6 dpi, which is in accordance with leaf phenotype development (Fig. 5C). To quantitatively assess tissue damage upon GFP-NbREM4 expression, electrolyte leakage from infiltrated leaves was measured at different time points. Ion leakage was significantly increased in GFP-NbREM4-expressing leaves compared with leaves transformed with the empty vector from 3 dpi onward (Fig. 5B), indicating that GFP-NbREM4 expression leads to membrane damage. To monitor GFP-NbREM4-triggered molecular changes, expression of selected defense related genes was measured $24 \mathrm{dpi}$ and $48 \mathrm{~h}$ postinfiltration (hpi), before any phenotypic changes became visible. The genes Pti5, Acre31, and Gras 2 have been demonstrated previously to be responsive to both a nonadapted bacterium and a semivirulent bacterial pathogen in $N$. benthamiana and, thus, have been implicated in PTI (Nguyen et al. 2010). Expression of Pti5 and Acre31 was induced in GFP-NbREM4-expressing plants at 48 hpi (Fig. 6). For Gras2, expression was higher at 24 hpi compared with $48 \mathrm{hpi}$, whereas Acre31 expression was maintained at similar levels between the two time points. Although not significantly upregulated, Gras 2 expression was increased by trend at $48 \mathrm{hpi}$ in GFP-NbREM4-infiltrated leaves (Fig. 6). In addition, GFPNbREM4 overexpression led to the significant upregulation of the pathogenesis-related genes Hin1 and Hsr201 (Fig. 6). Taken together, transient overexpression of NbREM4 in leaves of $N$. benthamiana induces expression of immunity-related genes $48 \mathrm{hpi}$ and leads to tissue collapse at later time points.

NbREM4 interacts with the immune-related kinase PBS1.

It has been hypothesized that certain remorin isoforms play a dynamic role as scaffold proteins in plant innate immune signaling (Benschop et al. 2007; Jarsch and Ott 2011). Thus, we sought to identify plant proteins capable of interacting with NbREM4 which could provide further information about NbREM4 function. To this end, a $\mathrm{Y} 2 \mathrm{H}$ screening of a tobacco cDNA library using NbREM4 as a bait was conducted. This identified a PBS1-like protein as a potential NbREM4
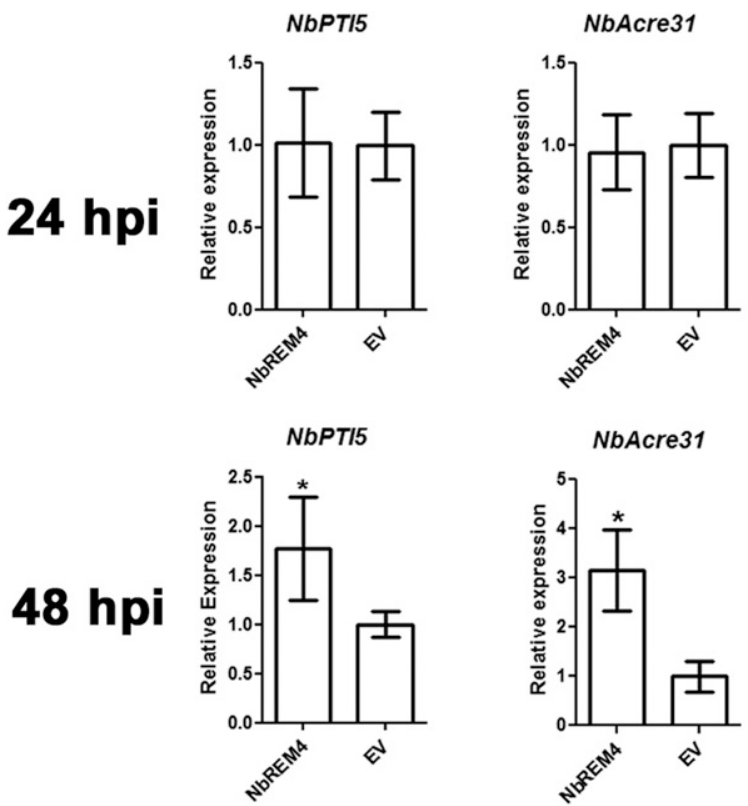

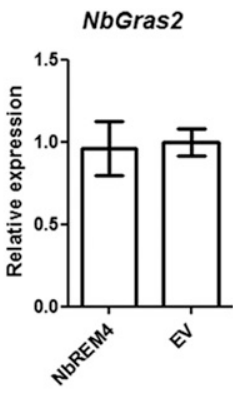

NbGras2

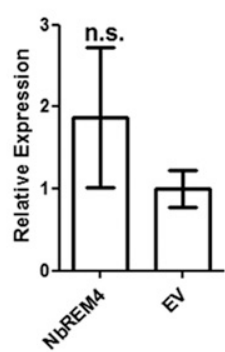

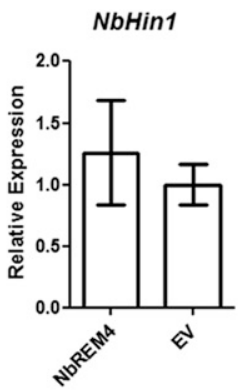

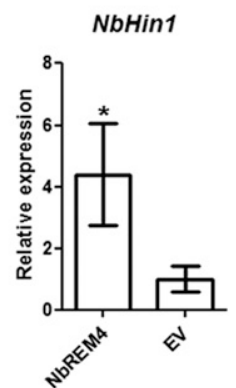

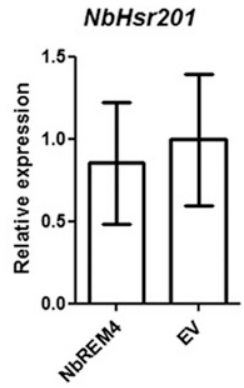

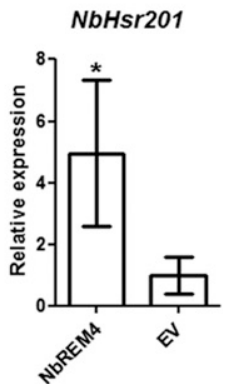

Fig. 6. Expression of selected defense-related genes in $N$. benthamiana leaves transiently expressing NbREM4. Quantitative real-time PCR of indicated defense-related genes was carried out on samples taken from $N$. benthamiana leaves transiently expressing NbREM4 or the empty vector control (EV) at the time points indicated; hpi $=$ hours postinfiltration. Actin was used as a reference gene. Each bar represents the mean of four biological replicates \pm standard error, and an asterisk $\left(^{*}\right)$ marks significant differences $(P>0.05)$ according to Student's $t$ test. 
interactor in yeast, which encodes an RLCK. PBS1-like proteins belong to subfamily VII of RLCKs that include the founding member PBS1 (Shao et al. 2003) and the Botrytisinduced kinase 1 (Laluk et al. 2011), as well as other PBS-like (PBL) proteins implicated in plant immune signaling (Lin et al. 2013; Rao et al. 2018).

We isolated a full-length sequence of the NbREM4-interacting PBL protein from N. benthamiana (Niben101Scf02086g00004.1). The protein comprises 390 amino acids and shares $71 \%$ similarity with PBS1 from Arabidopsis (Supplementary Fig. S6). Just as for AtPBS1, the $N$. benthamiana PBL protein carries a putative myristoylation signal at its $\mathrm{N}$ terminus which likely mediates PM

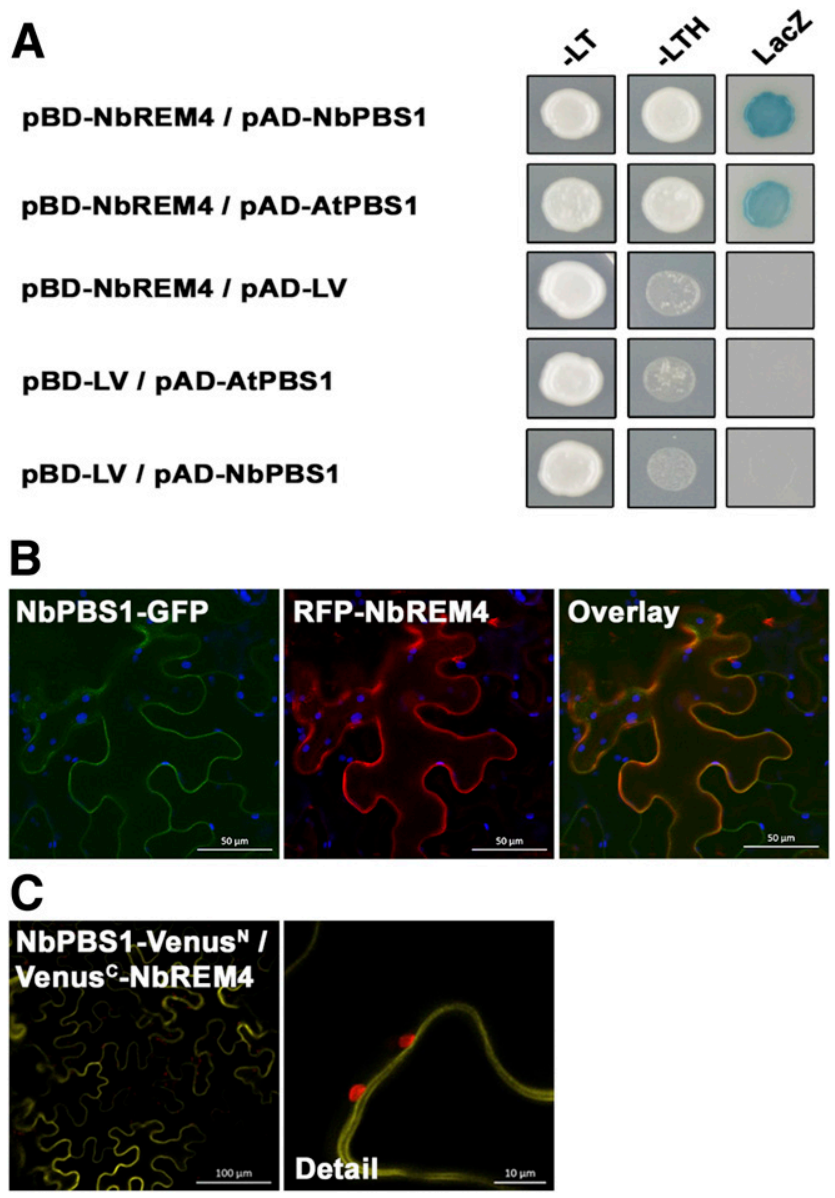

Fig. 7. NbREM4 interacts with PBS1 in yeast two-hybrid assays and in planta. A, NbREM4 fused to the GAL4 DNA binding domain (BD) was expressed in combination with PBS1 fused to the GAL4 activation domain (AD) in yeast strain Y190. Cells were grown on selective media before a LacZ filter assay was performed. The empty BD vector or AD vector served as a negative control. NbPBS1 = Nicotiana benthamiana PBS1; AtPBS1 = Arabidopsis thaliana PBS1; $-\mathrm{LT}=$ yeast growth on medium without Leu and Trp; -HLT = yeast growth on medium lacking His, Leu, and Trp, indicating expression of the HIS3 reporter gene; and LacZ $=$ activity of the lac $Z$ reporter gene. $\mathbf{B}$, Colocalization of NbPBS1-green fluorescent protein (GFP) and red fluorescent protein (RFP)-NbREM4 in N. benthamiana leaf epidermal cells. NbPBS1-GFP was coexpressed with RFP-NbREM4 by Agrobacterium infiltration. The green fluorescence (GFP), red fluorescence (mCherry), and chlorophyll autofluorescence were monitored separately to prevent cross-talk of the fluorescence channels and the resulting fluorescence images were merged. $\mathbf{C}$, Bimolecular fluorescence complementation in planta interaction of NbPBS1 with NbREM4. Yellow fluorescent protein (YFP) confocal microscopy images show $N$. benthamiana leaf epidermal cells transiently expressing NbPBS1-Venus ${ }^{\mathrm{N}}$ in combination with Venus ${ }^{\mathrm{C}}$ NbREM4. A close-up of the same cells shows that the YFP fluorescence produced by the interaction of NbPBS1-Venus ${ }^{\mathrm{N}}$ with Venus ${ }^{\mathrm{C}}$-NbREM4 aligns with the plasma membrane. association and contains a single catalytic kinase domain. Based on these analogies, we termed the $N$. benthamiana PBL protein $\mathrm{NbPBS} 1$. A direct $\mathrm{Y} 2 \mathrm{H}$ assay revealed that full-length $\mathrm{NbPBS} 1$ as well as Arabidopsis (At)PBS1 is capable of interacting with NbREM4 in yeast (Fig. 7A). In order to investigate whether NbREM4 specifically interacts with PBS1 or also with other members of the PBL family, we tested binding of the remorin to other RLCKs from Arabidopsis. NbREM4 interacted with AtPBS1 but not with any other RLCK tested, indicating a certain degree of specificity in the interaction of both proteins (Supplementary Fig. S7).

To assess the extent of colocalization of NbREM4 and PBS1 inside plant cells, NbREM4 N-terminally tagged with the red fluorescent protein and NbPBS1-GFP were coexpressed in leaves of $N$. benthamiana using Agrobacterium infiltration. Confocal imaging $2 \mathrm{dpi}$ revealed that green as well as red fluorescence aligned with the region representing the PM and both fluorescence signals displayed substantial overlap, indicative of colocalization of both proteins (Fig. 7B). We applied the BiFC approach to analyze whether the observed $\mathrm{Y} 2 \mathrm{H}$ interactions occur in living plant tissues or cells. Transient coexpression of NbPBS1-Venus ${ }^{\mathrm{N}}$ together with Venus ${ }^{\mathrm{C}}$ NbREM4 induced a strong fluorescence signal along the PM not engulfing the chloroplasts (Fig. 7C). Control experiments indicate that the BiFC signal is specific. In summary, these experiments suggest that NbREM4 specifically interacts with PBS1 at the PM of plant cells.

\section{PBS1 phosphorylates NbREM4 in vitro.}

Arabidopsis PBS1 belongs to subgroup VII of RLCK, of which several members have been shown to play redundant roles in mediating PTI responses upon phosphorylation by upstream PRRs (Bi et al. 2018; Rao et al. 2018; Yamada et al. 2016). Based on protein mobility shifts in sodium dodecyl sulfate polyacrylamide gel electrophoresis (SDS-PAGE), PBS1 has been shown to undergo flg22-dependent phosphorylation in Arabidopsis protoplasts, suggesting an activation of the kinase during PTI signaling (Lu et al. 2010; Zhang et al. 2010). However, downstream phosphorylation targets of PBS1 have not been identified thus far. Given that PBS1 interacts with NbREM4 and that remorins have been described as phosphoproteins (Benschop et al. 2007; Marín and Ott 2012; Reymond et al. 1996; Tóth et al. 2012), we explored the possibility of NbREM4 being a phosphorylation substrate of PBS1. To this end, both proteins were recombinantly produced in Escherichia coli and subjected to an in vitro phosphorylation assay using radiolabeled ATP. As indicated by their autophosphorylation activity, both NbPBS1 and AtPBS1 recombinant proteins appeared to be active in vitro (Fig. 8A). Under our experimental conditions AtPBS1 reproducibly produced a stronger autophosphorylation signal than NbPBS1, indicating higher activity of the recombinant protein from Arabidopsis. However, when either of the two PBS1 proteins (NbPBS1 or AtPBS1) was present in the same assay mix as MBP-NbREM4, an additional signal appeared that corresponds to the MBP-NbREM4 protein band, indicating phosphorylation of NbREM4 by PBS1 in vitro (Fig. 8A). The addition of MBP alone produced no additional signal, demonstrating phosphorylation of the NbREM4 portion within the MBP-NbREM4 fusion protein.

The larger difference in molecular weight between AtPBS1 and NbREM4 as opposed to NbPBS1 and NbREM4 resulted in a better separation of the autophosporylation and transphosphorylation signals. Thus, we used AtPBS1 for subsequent in vitro phosphorylation experiments involving NbREM4. In order to map NbREM4 phosphorylation sites, phosphorylation reactions were repeated under nonradioactive conditions and protein from gel-excised MBP-NbREM4 bands was subjected 

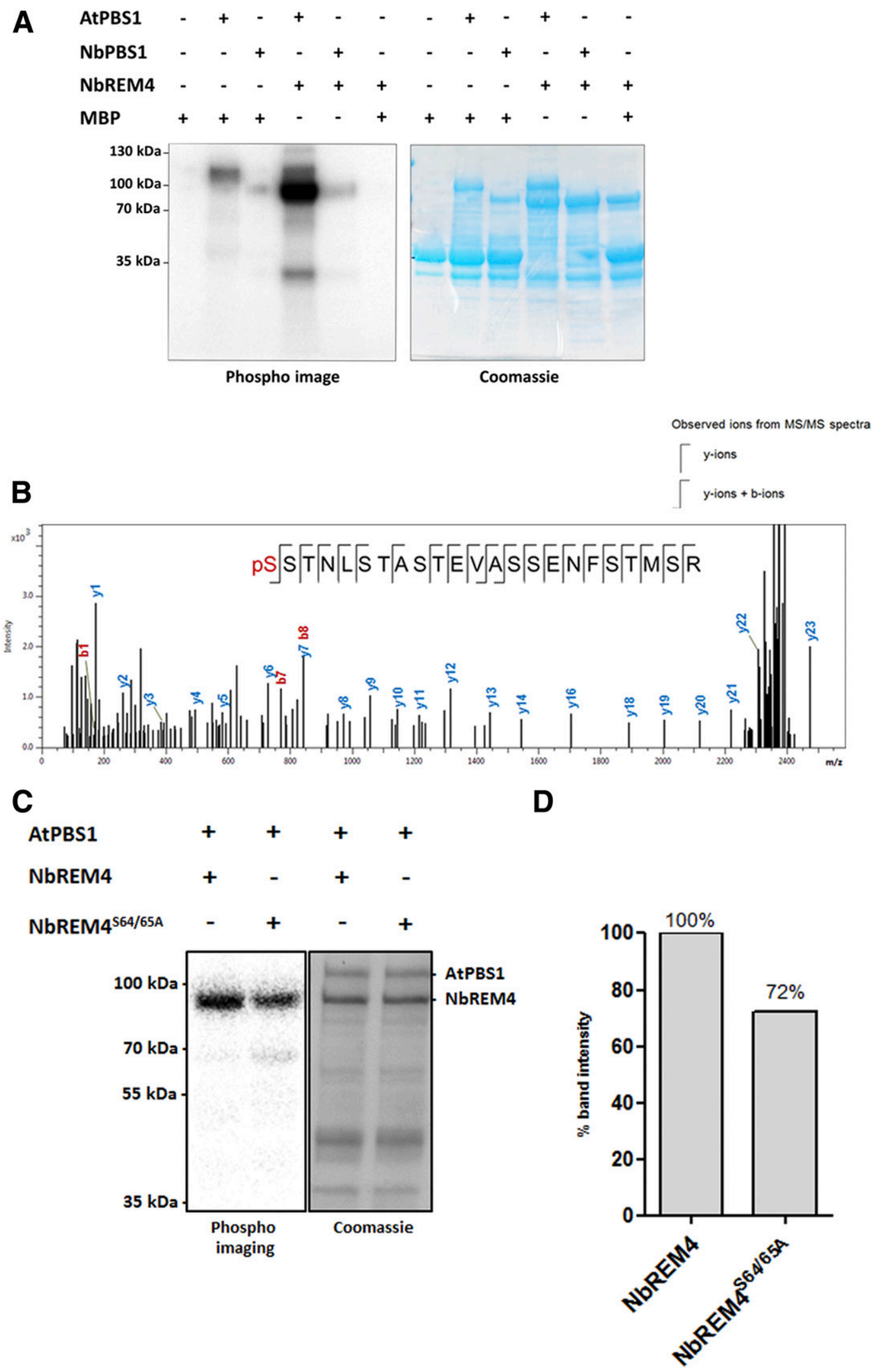

Fig. 8. PBS1 phosphorylates NbREM4 in vitro. A, In vitro phosphorylation assay of NbREM4 by AtPBS1. Recombinant proteins were incubated in the presence of 32P-ATP and the mixture was subsequently resolved by sodium dodecyl sulfate polyacrylamide gel electrophoresis (SDS-PAGE). Maltose-binding protein $(\mathrm{MBP})$ served as a negative control. Left $=$ autoradiogram and right $=$ Coomassie blue stain for protein visualization. B, Matrix-assisted laser desorption-ionization time of flight tandem mass spectrometry spectrum of an ion at $\mathrm{m} / \mathrm{z} 2474.03$ derived from in vitro phosphorylated NbREM4. Peptide fragmentation provides strong evidence for the sequence 64S to 86R of the NbREM4 polypeptide with phosphorylation occurring at 64S (inset). Intact $\mathrm{y}$-ions and b-ions are labeled with $\mathrm{y}$ and $\mathrm{b}$, respectively. C, In vitro phosphorylation of NbREM $4^{\mathrm{S} 64 / 65 \mathrm{~A}}$ compared with the wild type NbREM4 protein. Recombinant proteins were incubated in the presence of 32P-ATP and the mixture was subsequently resolved by SDS-PAGE. Left $=$ autoradiogram and right $=$ Coomassie blue stain for protein visualization. D, Densitometric analysis of NbREM4 ${ }^{\mathrm{S} 64 / 65 \mathrm{~A}}$ phosphorylation using ImageJ. 
to matrix-assisted laser desorption-ionization time of flight (MALDI-TOF) tandem mass spectrometry (MS/MS) analysis. This identified a singly phosphorylated peptide comprising the amino acids 64 to 87 of the NbRemorin4 protein (relative to the start $\mathrm{M}$ of the native NbREM4 protein) (Fig. 8B). The peptide contained several serine and threonine residues that potentially could serve as phosphoacceptor sites. Fragmentation analysis suggested phosphorylation at one of the N-terminal residues of the phosphopeptide located within the double serine motif S64 (62\% probability) or S65 (35\% probability). We created an MBP-NbREM4 $4^{\mathrm{S} 64 / 65 \mathrm{~A}}$ variant that carries a serine to alanine substitution at the potential phosphorylation site and used the purified recombinant protein in an in vitro kinase assay with AtPBS1. This still yielded a signal corresponding to MBPNbREM $4{ }^{\mathrm{S} 64 / 65 \mathrm{~A}}$, indicating that the serine to alanine substitution at this position did not abolish phosphorylation (Fig. 8C). However, a densitometric analysis of signal intensity revealed a reduction of phosphorylation by approximately $30 \%$ (Fig. 8D). This suggests that phosphorylation of NbREM4 by AtPBS1 at either S64 or S65 occurs but that the protein contains additional phosphorylation sites that escaped the MALDI-TOF MS/MS analysis. The presence of additional phosphosites in NbREM4 is in accordance with an in silico phosphosite prediction using NetPhos3.1, which predicts at least 28 putative serine or threonine phosphorylation sites with high confidence (Supplementary Fig. S8).

Taken together, these data suggest that PBS1 phosphorylates NbREM4 at multiple sites in vitro and, thus, opens the possibility that NbREM4 is also a phosphorylation target of PBS1 in planta.

\section{NbREM4 is not acetylated by HopZ1a in vitro.}

HopZ1a possess acetyltransferase activity, and acetylation of target proteins has been associated with virulence and avirulence functions of the effector (Jiang et al. 2013; Lee et al. 2012; Lewis et al. 2013). In order to investigate whether the
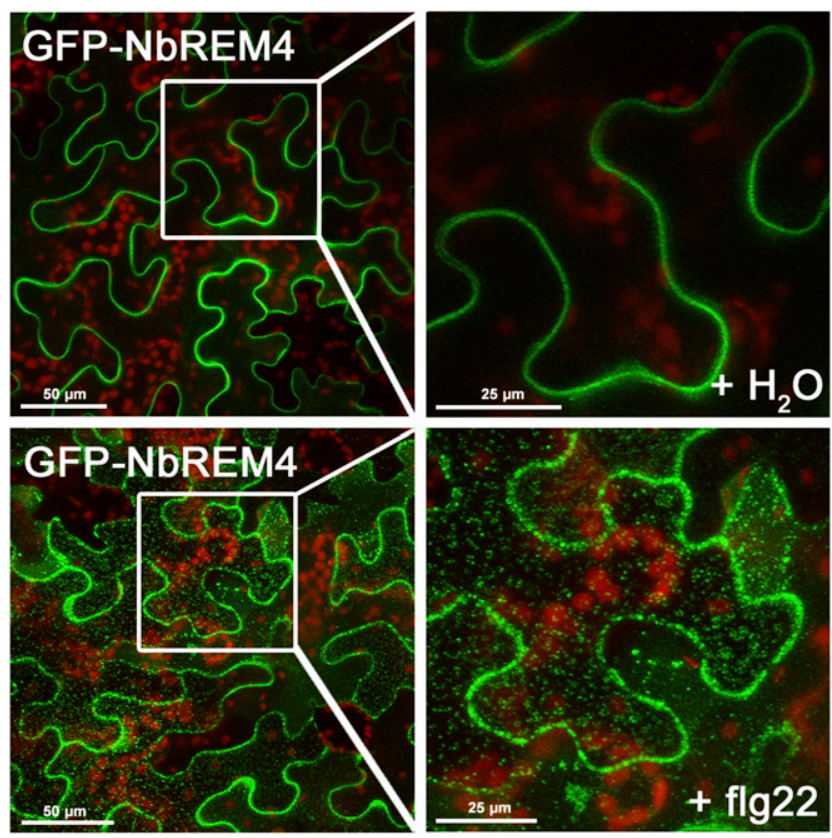

Fig. 9. Relocation of NbREM4 upon flg22 treatment into punctuate structures. Confocal micrographs of Nicotiana benthamiana leaf epidermal cells transiently expressing green fluorescent protein (GFP)-NbREM4 challenged flg22 as compared with an $\mathrm{H}_{2} \mathrm{O}$ control. The flg 22 treatment was performed $48 \mathrm{~h}$ postinfiltration and $60 \mathrm{~min}$ before imaging. Left $=$ overview and right $=$ magnification of the inset indicated on the left.
HopZ1a interaction partner NbREM4 also serves as an acetylation target, an in vitro acetylation assay was conducted using E. coli-produced proteins. Purified recombinant proteins were incubated with ${ }^{14} \mathrm{C}$-acetyl-coenzyme $\mathrm{A}$ and $100 \mathrm{nM}$ inositol-hexakisphosphate (IP6) for $1 \mathrm{~h}$ at $30^{\circ} \mathrm{C}$. Subsequently, the proteins were separated by SDS-PAGE analysis followed by autoradiography. IP6 is a eukaryotic cofactor that stimulates the acetyltransferase activity of effectors in the YopJ family, including HopZ1a (Lee et al. 2012).

Autoacetylation of MBP-HopZ1a was readily detected, indicating enzymatic activity of the recombinant protein (Supplementary Fig. S9). However, no clear signs of an acetylation of the GST-NbREM4 band could be detected, suggesting that the remorin does not constitute an acetylation target of HopZ1a in vitro.

\section{NbREM4 relocates to PM nanodomains after flg22 treatment.}

Previous reports have localized individual remorin isoforms from different plant species to PM nanodomains in either a constitutive or stimulus-dependent manner (Demir et al. 2013; Jarsch et al. 2014; Lefebvre et al. 2010; Ott 2017; Raffaele et al. 2009). Transient expression of GFP-NbREM4 in leaves of $N$. benthamiana did not reveal clear signs of localization to distinct membrane subdomains (Fig. 9). Given the fact that NbREM4 could potentially be involved in PAMP signaling at the PM, we investigated whether flg22 treatment would have any influence on GFP-NbREM4 PM localization. Indeed, we could observe that, $1 \mathrm{~h}$ after treatment with flg22, GFPNbREM4 fluorescence was increasingly found in distinct and immobile nanodomains that appeared to be associated with the PM leaflet of treated cells (Fig. 9). Imaging of the upper surface plane of flg22-treated GFP-NbREM4-expressing cells as compared with cells without flg22 treatment further supports the notion that GFP-NbREM4 localizes to PM nanodomains after flg22 treatment (Supplementary Fig. S10).

\section{Downregulation of NbREM4 does not affect HopZ1a-dependent ETI.}

HopZ1a has been shown to elicit an HR in N. benthamiana, indicative of its recognition through an $\mathrm{R}$ protein (Ma et al. 2006). However, Baudin et al. (2017) recently found that inducible expression of HopZ1a in $N$. benthamiana did not induce an HR. In Arabidopsis, HopZ1a recognition requires the canonical CC-type NB-LRR receptor (NLR) protein ZAR1 (Lewis et al. 2010). Detection of HopZ1a by AtZAR1 requires AtZED1, an RLCK belonging to the RLCK clade XII-2 family that acts as decoy guarded by AtZAR1 to sense the activity of HopZ1a (Lewis et al. 2013). Given the discrepancy in the observed HopZ1a overexpression phenotypes, we initiated a set of experiments to further investigate an HopZ1a-dependent HR in $N$. benthamiana and explore the possible involvement of NbREM4 and PBS1 in this process. First, we assessed whether, under our experimental conditions, expression of HopZ1a leads to HR development in $N$. benthamiana. To this end, HopZ1a, HopZ1 $\mathrm{a}^{\mathrm{C} / \mathrm{A}}$ and the $X$. campestris pv. vesicatoria T3E AvrRxv were transiently expressed in $N$. benthamiana leaves alongside an empty vector (EV) control. As compared with the EV control and the catalytically inactive HopZ1a ${ }^{\mathrm{C} / \mathrm{A}}$ variant, HopZ1ainfiltrated areas showed clear signs of cell death at 2 dpi that were even more prominent than those triggered by AvrRxv (Fig. 10A). In accordance with the observed leaf phenotype, ion leakage from HopZ1a-infiltrated tissue was significantly increased at 2 dpi whereas expression of HopZ1 ${ }^{\mathrm{C} / \mathrm{A}}$ did not increase electrolyte efflux (Fig. 10B). A Western blot analysis confirmed expression of all proteins tested (Fig. 10C). Thus, transient expression using Agrobacterium infiltration triggered 
HR-like symptoms and membrane damage in $N$. benthamiana. In order to investigate whether the observed HopZ1a-dependent phenotype would require the $\mathrm{R}$ protein ZAR1, we cloned a fragment of the $N$. benthamiana ZARl ortholog (Baudin et al. 2017) into a vector for virus-induced gene silencing (VIGS). Strong downregulation of NbZARl could be confirmed in $N$. benthamiana plants 2 weeks after infiltration with the VIGS vectors as compared with the GFP-VIGS control (Fig. 11A). Transient expression of HopZ1a in GFP-VIGS plants led to the previously observed HR symptoms at 2 dpi whereas HopZ1 $\mathrm{a}^{\mathrm{C} / \mathrm{A}}$ did not cause phenotypic changes (Fig. 11B). In contrast, HopZ1a expression in NbZARI-VIGS plants did not yield visible signs of $\mathrm{HR}$ at $2 \mathrm{dpi}$ (Fig. 11B). Protein expression in all tissues analyzed was confirmed by Western blotting (Fig. 11C). Ion leakage measurement revealed that HopZ1a did not cause a significant increase in membrane damage in NbZAR1VIGS plants as opposed to the GFP-VIGS control (Fig. 11D). Taken together, the data suggest that, similar to the situation in Arabidopsis (Lewis et al. 2010), the HopZ1a-dependent HR in $N$. benthamiana depends on its recognition by NbZAR1.

We next sought to explore whether the HopZ1a interaction partner NbREM4 contributes to HopZ1a-asssociated HR in $N$. benthamiana. Therefore, we used the VIGS system to repress NbREM4 expression and subsequently expressed HopZ1a in NbREM4-silenced plants. NbREM4 expression was drastically reduced in NbREM4-VIGS plants, indicating efficient silencing (Supplementary Fig. S11). However, NbREM4VIGS plants displayed HR symptoms and electrolyte leakage upon HopZ1a expression similar to the GFP-VIGS control, suggesting that downregulation of NbREM4 does not affect the ability of HopZ1a to elicit an HR.

\section{DISCUSSION}

During infection, bacterial T3Es translocated by the type III secretion system play a central role in the manipulation of the host cellular machinery in favor of the pathogen. In general, these T3Es are essential for pathogen virulence by interfering with plant processes involved in defense responses (Jones and Dangl 2006). In order to interact with the correct host target and exert their function, T3Es often show a specific localization within the host cell (Hicks and Galán 2013). A recent survey suggests that, in the case of $P$. syringae, more than half of the T3Es characterized to date target host proteins that localize to the host cell's PM (Khan et al. 2018a). This highlights the importance of the PM as the interface of pathogen recognition and initiation of immunity (Hoefle and Hückelhoven 2008). Accordingly, a number of effector proteins carry lipid modifications such as acylation (also called palmitoylation), myristoylation, and prenylation that enable host membrane association after translocation into the cytosol (Hicks and Galán 2013). The $P$. syringae T3E HopZ1a is an acetyltransferase that requires $\mathrm{N}$-terminal myristoylation for PM targeting, and this modification is required for its virulence and avirulence function in Arabidopsis (Lewis et al. 2008) and soybean (Zhou et al. 2009). Several distinct molecular targets of HopZ1a have been identified, not all of which are known to locate to the PM (Jiang et al. 2013; Lee et al. 2012; Zhou et al. 2011). However, HopZ1a has been shown to interfere with PM- and cell-wall-associated defense such as callose deposition, the ROS burst, and MAP kinase activation (Lee et al. 2012; Lewis et al. 2014). This is partially explained by its ability to cause microtubule destruction through acetylation of tubulin (Lee et al. 2012).

Here, we identified the remorin protein NbREM4 from $N$. benthamiana as a novel interaction partner of HopZ1a in plants. The obtained evidence suggests that the HopZ1a-NbREM4 interaction occurs at the PM and does not require additional proteins.
Remorins are plant-specific proteins with multiple roles in plant-microbe interactions, including those with viruses ( $\mathrm{Fu}$ et al. 2018; Perraki et al. 2014; Raffaele et al. 2009; Son et al. 2014), oomycetes (Bozkurt et al. 2014), and bacteria (Lefebvre et al. 2010; Liang et al. 2018; Tóth et al. 2012). Interestingly, the Arabidopsis remorins AtREM1.2 and AtREM1.3 have been identified to interact with the Pseudomonas T3E HopF2 in vivo by two independent methods (Hurley et al. 2014; Khan et al. 2018b). Although the functional significance of this interaction is currently unknown, it might point to broader role of remorins for T3E functioning in plants. All described remorins associate with the $\mathrm{PM}$ in dynamic subdomains recently classified as nanodomains,
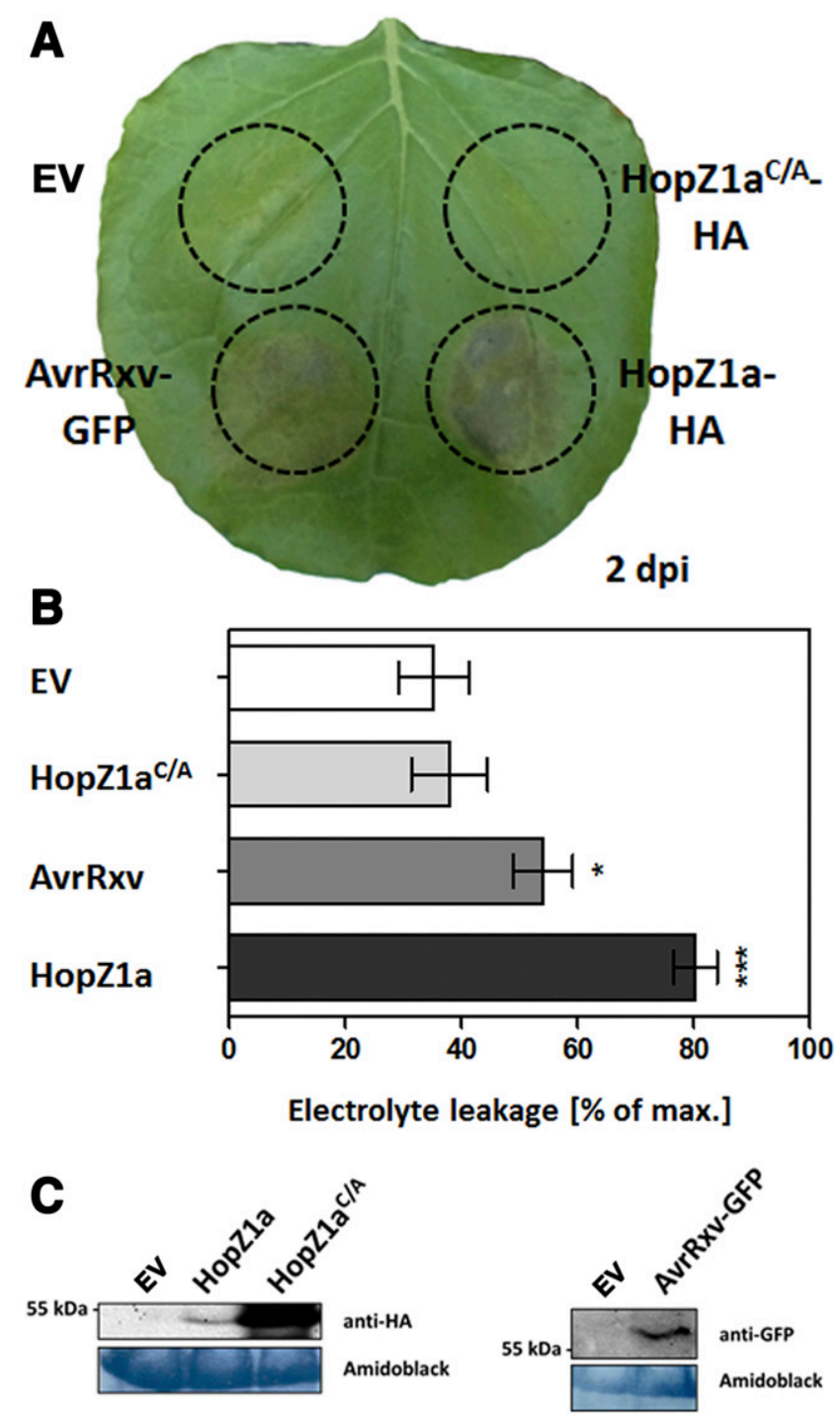

Fig. 10. Transient HopZ1a expression induces an hypersensitive response in Nicotiana benthamiana leaves. A, Agrobacterium-mediated transient expression assay in $N$. benthamiana. Constructs indicated were inoculated into the leaf areas delineated by the dashed line. The picture was taken $48 \mathrm{~h}$ postinfiltration (hpi). EV = empty vector, GFP = green fluorescent protein, $\mathrm{HA}=$ hemagglutinin, and dpi = days postinfiltration. B, Ion leakage was measured in plants transiently expressing the constructs indicated $48 \mathrm{hpi}$ with agrobacteria. Bars represent the average ion leakage measured for triplicates of six leaf disks each, and the error bars indicate standard deviation. Asterisks indicate a significant difference (** and *** indicate $P<$ 0.05 and 0.01 , respectively) based on results of a Student's $t$ test. C, Protein immunoblot of HopZ1a-HA, HopZ1 ${ }^{\text {C/A }}$-HA, and AvrRxv-GFP, verifying protein expression. Samples were collected $48 \mathrm{hpi}$ and probed with $\alpha-\mathrm{HA}$ antibody or $\alpha$-GFP antibody. Amido-black staining is included to show equal loading of the samples. 
which are assumed to constitute assembly platforms for signal transduction, pathogen infection, and other processes (Gronnier et al. 2017; Jarsch and Ott 2011; Jarsch et al. 2014; Ott 2017) . It has been shown that Medicago truncatula nodulation-induced remorin MtSYMREM1 interacts with at least three receptor-like kinases (NFP, LYK3, and DMI) that are essential for root nodule symbiosis (Lefebvre et al. 2010). Infection-dependent induction of MtSYMREM1 results in recruitment of ligand-activated LYK3 and its stabilization within nanodomains to prevent endocytosis of the receptor, a function required for successful rhizobial infection (Liang et al. 2018). We observed relocalization of GFP-NbREM4 to distinct PM subdomains resembling nanodomains after treatment with flg22. A similar redistribution into specific PM subdomains upon elicitation with flg22 has also been observed for the flg22 receptor FLS2 (Keinath et al. 2010). This supports a model in which PAMP-induced signaling may require defined membrane nanodomains that provide physical rafts for receptor-scaffold and other protein-protein interactions, possibly involving NbREM4. Phosphorylation could be one mechanism which regulates membrane sublocalization or protein-protein interactions of NbREM4. Many remorins have been shown to be phosphorylated in a constitutive or stimulus-dependent manner (Jarsch and Ott 2011). In a large-scale proteomic approach to identify differentially phosphorylated proteins involved in early PAMP, AtREM1.3 was shown to be phosphorylated in an flg22-dependent manner while other remorin isoforms displayed constitutive phosphorylation (Benschop et al. 2007). The potato remorin StREM1.3 is differentially phosphorylated by a PM-associated protein kinase upon the perception of polygalacturonic acid (Jarsch and Ott 2011; Reymond et al. 1996). Generally, phosphorylation of remorins appears to be restricted to serine and threonine residues that are exclusively located within the lowly conserved $\mathrm{N}$-terminal region of the protein (Marín and Ott 2012). The N-terminal domain of remorins is intrinsically disordered, and phosphorylation within this region has the capacity to alter the interaction of remorins with other proteins (Marín et al. 2012). We could show that NbREM4 is phosphorylated on several residues, including serine 64 within the unconserved N-terminal domain, by the PM-localized RLCK PBS1 from $N$. benthamiana as well as by the heterologous protein from Arabidopsis in vitro. PBS1 belongs to subfamily VII of RLCKs whose members have been implicated in several aspects of PTI, including activation of MAP signaling, generation of ROS, and transcriptional reprogramming (Bi et al. 2018; Lal et al. 2018; Lin et al. 2013; Rao et al. 2018).

The role of PBS1 in PTI signaling is not entirely clear. It has been shown that flg22 treatment induces phosphorylation of PBS1 in Arabidopsis as well as in wheat (Lu et al. 2010; Sun et al. 2017). However, genetic studies indicate that, at least in Arabidopsis, PBS1 plays only a minor role PTI signaling as compared with other members of the RLCK VII family (Zhang
A

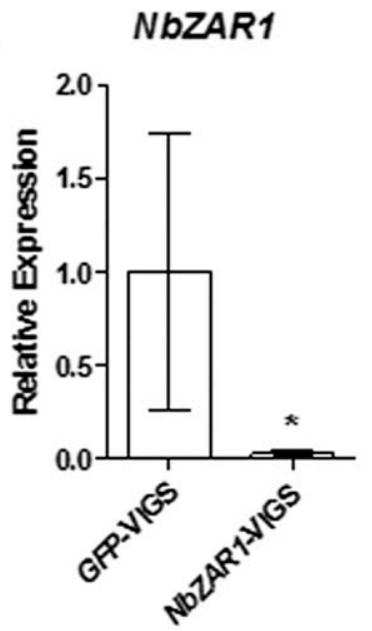

C

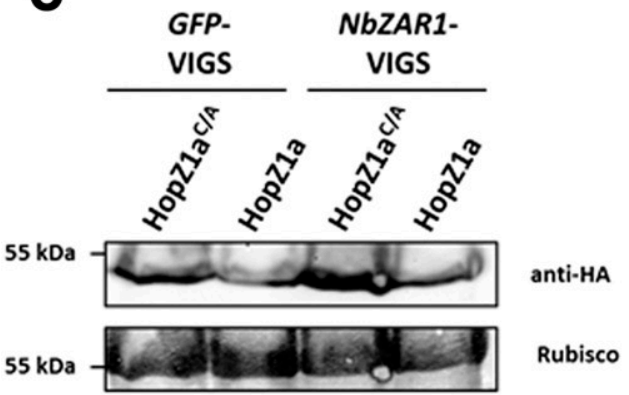

B
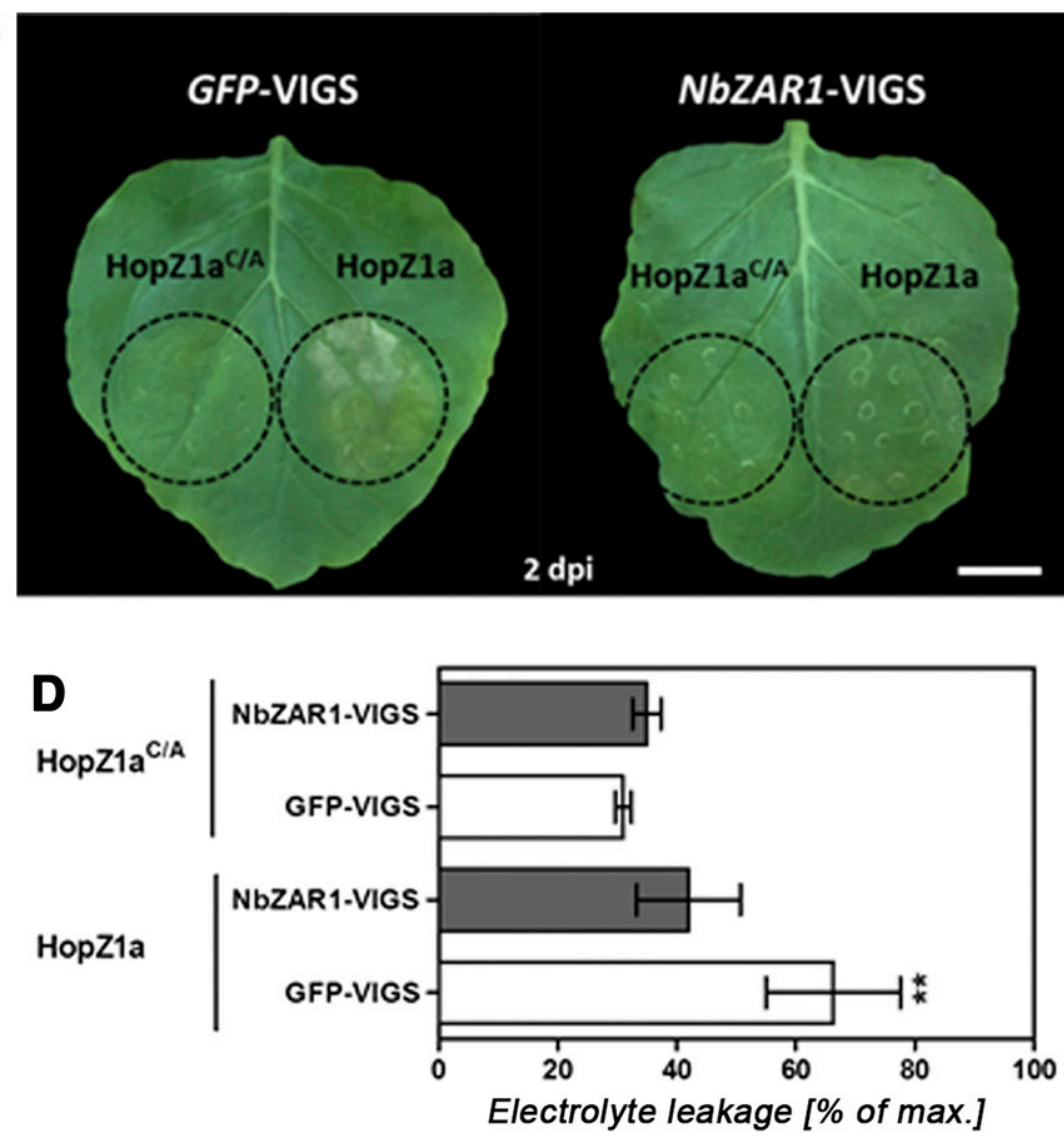

Fig. 11. Downregulation of ZAR1 expression abolishes HopZ1a-triggered hypersensitive response. A, Quantitative real-time PCR (qRT-PCR) analysis of ZAR1 downregulation in NbZAR1-virus-induced gene silencing (VIGS) Nicotiana benthamiana plants. Total RNA was isolated from leaves of VIGS plants 14 days postinfiltration (dpi) and subjected to cDNA synthesis followed by qRT-PCR. Bars represent the mean of at least three biological replicates \pm standard deviation. GFP $=$ green fluorescent protein. The asterisk $(*)$ indicates a significant difference $(P<0.05)$ based on results of a Student's $t$ test. B, Leaves of NbZAR1-VIGS and GFP-VIGS plants were infiltrated with the constructs indicated by the dashed line. C, Protein immunoblot of HopZ1a-hemagglutinin (HA) and HopZ1 ${ }^{\mathrm{ClA}}-\mathrm{HA}$, verifying protein expression of VIGS plants. Samples were collected 2 dpi and probed with an $\alpha$-HA antibody. Amido-black staining is included to show equal protein loading. D, Ion leakage was measured in VIGS plants transiently expressing the constructs indicated on the left $48 \mathrm{~h}$ postinfiltration. Bars represent the average ion leakage measured for triplicates of six leaf discs each, and the error bars indicate standard deviation. Asterisks $(* *)$ indicate a significant difference $(P<0.01)$ based on results of a Student's $t$ test. 
et al. 2010). Whether PBS1 in other plants is more important to trigger PAMP-induced defense responses is currently unknown. NbREM4 does not seem to interact with tested members of the Arabidopsis RLCK VII clade in yeast other than PBS1. However, because we did not test additional RLCK family members from $N$. benthamiana, this does not exclude phosphorylation of NbREM4 by other kinases in vivo. Phosphorylation of NbREM4 by an upstream RLCK such as PBS1 could then lead to activation of downstream defense responses; for instance, by altering the ability of NbREM4 to interact with other proteins and thereby affecting their activity. Along that line, we could observe that transient overexpression of NbREM4 in leaves of $N$. benthamiana led to the development of leaf chlorosis associated with membrane damage and the induction of defense-related genes. Thus, overexpression of NbREM4 might dominantly activate downstream defense responses. However, we currently cannot exclude a general cytotoxic effect of NbREM4 through interference with other vital cellular processes.

The unequivocal demonstration that NbREM4 phosphorylation is required for relocalization of the protein into membrane nanodomains or that it alters protein-protein interactions of NbREM4 downstream immune-related targets requires further experimentation.

The virulence function of HopZ1a has been associated with its acetyltransferase activity, and acetylation of host target proteins by HopZ1a is thought to interfere with their function in immunity-related processes (Jiang et al. 2013; Lee et al. 2012; Zhou et al. 2011). The results of in vitro acetylation assays do not suggest acetylation of NbREM4 by HopZ1a and, thus, it is currently unknown whether NbREM4 constitutes a genuine effector target for HopZ1a or whether it might have some auxiliary function. Remorins have not been described as target proteins for bacterial T3Es thus far but, given their extensive involvement in plant-microbe interactions, interference with remorin function could potentially have a wide impact on PTI-related defense outputs. Alternatively, HopZ1a could use NbREM4 as an auxiliary factor that facilitates either the indirect interaction with other proteins or the localization to specific membrane subdomains related to HopZ1a function. RLCKs such as PBS1 could constitute these indirect targets and future experiments have to investigate whether PBS1 or other NbREM4-interacting proteins are substrates for HopZ1a acetylation and, hence, whether interaction of HopZ1a with NbREM4 is related to the virulence function of the effector.

In Arabidopsis, HopZ1a is recognized by the NLR ZAR1 to trigger ETI. Recognition depends on the pseudokinase ZED1, which belongs to the RLCK family XII and acts as a decoy for HopZ1a-mediated acetylation to activate ZAR1 (Lewis et al. 2013). Recent evidence suggests that the ZAR1 immune pathway is largely conserved in $N$. benthamiana (Baudin et al. 2017). Our observation that transient expression of HopZ1a in $N$. benthamiana triggers an ETI response is in line with previous reports (Ma et al. 2006) but in contrast to a recent study by Baudin et al. (2017), who observed an HopZ1a-dependent HR in $N$. benthamiana only upon coexpression of the effector with ZED1 from Arabidopsis. The discrepancy between these two observations is likely due to differences in the experimental setup. Although in the work of Ma et al. (2006) and the study described here a strong constitutive CaMV35S promoter was used to drive HopZ1a expression in $N$. benthamiana leaves, Baudin et al. (2017) used an inducible system that might lead to differences in expression kinetics and protein amounts of the effector and, thus, could affect the outcome of the overexpression. As previously observed (Baudin et al. 2017), silencing of ZAR1 abolished the HopZ1a-triggered HR in $N$. benthamiana, confirming effector recognition by this NLR also in this species. Based on the current model of HopZ1a recognition by ZAR1 in Arabidopsis, this implies that HopZ1a likely interacts with and modifies a protein that is guarded by ZAR1 in N. benthamiana. A ZED1 ortholog in N. benthamiana has not been characterized thus far and, therefore, potential guardees of NbZAR1 are currently unknown. Recent data suggest that ZAR1 in Arabidopsis interacts with multiple guardees beyond ZED1, including ZRK1, leading to the recognition of AvrAC from X. campestris pv. campestris (Wang et al. 2015) and ZRK3 required for the perception of HopF2a from $P$. syringae (Seto et al. 2017). Thus, it is possible that, in $N$. benthamiana, ZAR1 guards various members of the RLCK family. Schultink et al. (2019) identified four RLCK XII genes within the $N$. benthamiana genome, and silencing of one particular isoform named JIM2 compromised the ability of the plant to recognize the $X$. perforans T3E XopJ4. It is tempting to speculate that another member of this family could act as a decoy during recognition of HopZ1a; however, this requires further testing. In Arabidopsis, PBS1 is guarded by the NLR RPS5 which activates HR upon proteolytic cleavage of PBS1 by the $P$. syringae T3E AvrPphB (Shao et al. 2003), thus providing precedent for a role of PBS1 in ETI. Recognition of AvrAC in Arabidopsis requires PBS-like 2 (PBL2) in addition to ZRK1, and the current model suggests that ZAR1 forms a stable complex with ZRK1, which specifically recruits PBL2 when the latter is uridylylated by AvrAC to subsequently trigger ZAR1-mediated immunity (Wang et al. 2015). Thus, modification of PBL2 by AvrAC is indirectly recognized by the ZAR1/ZRK1 immune complex. Silencing of NbREM4 did not affect HopZ1a-triggered HR in $N$. benthamiana, arguing against a direct or indirect involvement of this with protein in HopZ1a recognition. In addition, the timing of phenotype development upon transient NbREM4 expression appears to be rather slow for ETI (Morel and Dangl 1997). This might suggest a more downstream involvement of NbREM4 in defense.

In summary, the present study shows that HopZ1a directly interacts with NbREM4 in $N$. benthamiana and transient overexpression of NbREM4 point toward a role of this remorin in induced defense responses. This notion is corroborated by the finding that NbREM4 interacts with and is phosphorylated by the immune-related RLCK PBS1. Future studies have to clarify whether the interaction with HopZ1a interferes with this possible role of NbREM4 in defense. VIGS studies indicate that neither NbREM4 nor PBS1 play a role in HopZ1atriggered ETI in $N$. benthamiana, favoring a role for these proteins as virulence targets for this T3E. The finding that HopZ1a triggers a ZAR1-dependent HR in N. benthamiana in the absence of the Arabidopsis decoy protein ZED1 further extends the observation concerning conservation of ZAR1mediated HopZ1a recognition in this species (Baudin et al. 2017).

\section{MATERIALS AND METHODS}

\section{Plant material and growth conditions.}

Tobacco plants ( $N$. benthamiana) were grown in soil in a climate chamber with daily watering and subjected to a cycle of $16 \mathrm{~h}$ of light and $8 \mathrm{~h}$ of darkness at 25 and $21^{\circ} \mathrm{C}$, respectively, with light at $300 \mu \mathrm{mol} \mathrm{m} \mathrm{m}^{-2} \mathrm{~s}^{-1}$ and $75 \%$ relative humidity.

\section{Y2H analysis.}

$\mathrm{Y} 2 \mathrm{H}$ techniques were performed according to the yeast protocols handbook and the Matchmaker GAL4 Two-hybrid System 3 manual (both Clontech) using the yeast reporter strains AH109 and Y187. The entire HopZ1 a coding region was amplified by PCR using the primers listed in Supplementary 
Table $\mathrm{S} 1$ and inserted in the pGBT-9 vector generating a fusion between the GAL4 DNA binding domain (BD). The yeast strain Y187 carrying the BD-HopZ1a construct was mated with AH109 cells pretransformed with a library derived from tobacco (N. tabacum) source leaves (Börnke 2005). Diploid cells were selected on medium lacking Leu, Trp, and His supplemented with $4 \mathrm{mM} 3$-aminotriazole. Cells growing on selective medium were further tested for activity of the lac $Z$ reporter gene using filter lift assays. Library plasmids from his3- or lacZ-positive clones were isolated from yeast cells and transformed into E. coli before sequencing of the cDNA inserts. Direct interaction of two proteins was investigated by cotransformation of the respective plasmids in the yeast strain AH109, followed by selection of transformants on medium lacking Leu and Trp at $30^{\circ} \mathrm{C}$ for 3 days and subsequent transfer to medium lacking Leu, Trp, and His for growth selection and lac $Z$ activity testing of interacting clones.

\section{Plasmid construction.}

To generate plasmids containing the corresponding gene of interest, the entire open reading frame was amplified by PCR from Arabidopsis cDNA. The resulting fragments were inserted into the pENTR-D/TOPO vector according to the manufacturer's instructions (Thermo) and verified by sequencing. For $\mathrm{Y} 2 \mathrm{H}$ analysis, fragments were recombined into Gatewaycompatible versions of the GAL4 DNA BD vector pGBT-9 and the activation domain vector pGAD424 (Clontech) using L/R-clonase (Thermo). To generate translational fusions between NbREM4 and the GFP, coding sequences were inserted into the vector pK7FWG2 (Karimi et al. 2002). Constructs for bimolecular complementation analysis are based on Gatewaycloning-compatible versions of pRB-C-Venus ${ }^{\mathrm{N} 173}$ and $\mathrm{pRB}-$ C-Venus ${ }^{\mathrm{C} 155}$.

\section{Agrobacterium infiltration.}

For infiltration of N. benthamiana leaves, Agrobacterium tumefaciens $\mathrm{C} 58 \mathrm{C} 1$ was infiltrated into the abaxial air space of 4- to 6-week-old plants, using a needleless 2-ml syringe. Agrobacteria were cultivated overnight at $28^{\circ} \mathrm{C}$ in the presence of appropriate antibiotics. The cultures were harvested by centrifugation, and the pellet was resuspended in sterile water to a final optical density at $600 \mathrm{~nm}$ of 1.0. The cells were used for the infiltration directly after resuspension. Infiltrated plants were further cultivated in the greenhouse with daily watering, and subjected to cycle of $16 \mathrm{~h}$ of light and $8 \mathrm{~h}$ of darkness at 25 and $21^{\circ} \mathrm{C}$, respectively, with light at $300 \mu \mathrm{mol} \mathrm{m} \mathrm{s}^{-2}$ and $75 \%$ relative humidity.

\section{In vitro pull down.}

Recombinant MBP-NbREM4 from E. coli (BL21 DE3; New England Biolabs) lysates was immobilized on amylose resins (New England Biolabs) and incubated for $1 \mathrm{~h}$ at $4^{\circ} \mathrm{C}$ with total protein lysates from cell expressing GST-HopZ1a or GSTNbPBS1. Proteins were eluted, and analyzed by immunoblotting using either anti-GST antibody (Sigma) or anti-MBP antibody (New England Biolabs).

\section{Western blotting.}

Leaf material was homogenized in SDS-PAGE loading buffer (100 mM Tris- $\mathrm{HCl}$ [pH 6.8], 9\% $\beta$-mercaptoethanol, $40 \%$ glycerol, $0.2 \%$ bromophenol blue, and $4 \%$ SDS) and, after heating for $10 \mathrm{~min}$ at $95^{\circ} \mathrm{C}$, subjected to gel electrophoresis. Separated proteins were transferred onto nitrocellulose membrane (Porablot; Machery and Nagel). Proteins were detected by an anti-hemagglutinin-peroxidase high-affinity antibody (Roche) or anti-GFP antibody (Roche) via chemiluminescence (GE Healthcare) using an myECL imager (Thermo).
BiFC.

Constructs were transformed into A. tumefaciens C58C1 and transiently expressed by Agrobacterium infiltration in $N$. benthamiana. The BiFC-induced YFP fluorescence was detected by confocal laser-scanning microscopy (LSM510; Zeiss) 48 hpi. The specimens were examined using the LD LCI Plan-Apochromat 253/0.8 water-immersion objective for detailed images with excitation using the argon laser (458- or 488-nm line for BiFC and chlorophyll autofluorescence, respectively). The emitted light passed the primary beamsplitting mirrors at 458 or $514 \mathrm{~nm}$ and was separated by a secondary beam splitter at $515 \mathrm{~nm}$. Fluorescence was detected with filter sets as follows: on channel 3,530 to 560 band pass; and on channel 1 , for red autofluorescence of chlorophyll.

\section{RNA extraction and quantitative real-time PCR.}

Total RNA was isolated from leaf material and then treated with RNase-free DNase to degrade any remaining DNA. Firststrand cDNA synthesis was performed from $2 \mu \mathrm{g}$ of total RNA using Revert-Aid reverse transcription (Thermo). For quantitative real- time PCR, the cDNAs were amplified using SensiFAST SYBR Lo-ROX Mix (Bioline) in the AriaMx Realtime PCR System (Agilent Technologies) as previously described (Arsova et al. 2010). At least three biological repeats and three technical repeats were used for each analysis. The transcript level was standardized based on cDNA amplification of NbActin as a reference. Statistical analysis was performed using Student's $t$ test.

\section{VIGS.}

VIGS in $N$. benthamiana was essentially carried out as described previously (Liu et al. 2002a,b). In brief, a fragment of $N$. benthamiana NbREM4 was amplified by PCR and cloned into pTRV2-Gateway using the Gateway recombination system (Invitrogen) as described. The plasmids were transformed into A. tumefaciens C58C1. A lower leaf of a 4-week-old $N$. benthamiana plant was coinfiltrated with a mixture of agrobacteria carrying either pTRV1 or pTRV2 containing the target sequence or a GFP-negative control fragment, as described previously (Liu et al. 2002b). Silenced plants were analyzed 14 dpi.

\section{Ion leakage measurements.}

For electrolyte leakage experiments, triplicates of $1.76 \mathrm{~cm}^{2}$ of infected leaf material were taken at different time points, as indicated. Leaf discs were placed on the bottom of a 15-ml tube. Deionized water $(8 \mathrm{ml})$ was added to each tube. After $4 \mathrm{~h}$ of incubation in a rotary shaker at room temperature, conductivity was determined with a conductometer. To measure the maximum conductivity of the entire sample, conductivity was determined after boiling the samples for $1 \mathrm{~h}$ (Stall et al. 1974).

\section{In vitro kinase assay.}

Purified MBP-NbREM4 or MBP-NbPBS1 $(2 \mu \mathrm{g})$ were incubated in $20 \mu \mathrm{l}$ of reaction buffer (10 mM N-2-hydroxyethylpiperazine- $N^{\prime}$-2-ethanesulfonic acid [HEPES] [pH 7.4], $2 \mathrm{mM} \mathrm{MgCl}{ }_{2}, 2 \mathrm{mM} \mathrm{MnCl}$, and $0.2 \mathrm{mM}$ dithiothreitol [DTT]) containing $2 \mu \mathrm{Ci}$ of $\left[\gamma-{ }^{32} \mathrm{P}\right]$ ATP (Hartmann Analytics). Reactions were incubated at $30^{\circ} \mathrm{C}$ for $1 \mathrm{~h}$. The reaction was stopped by adding $4 \times$ Laemmli buffer and separate on a 4-to-12\% polyacrylamide gel. The gel was stained with Coomassie blue and incorporated radiolabel was visualized by autoradiography.

\section{In vitro acetylation assay.}

Purified proteins $(2 \mu \mathrm{g})$ were incubated in $20 \mu \mathrm{l}$ of acetylation buffer (100 nM IP6, $50 \mathrm{mM}$ HEPES [pH 8.0], 10\% [vol/vol] glycerol, and $5 \mathrm{mM}$ DTT), supplemented with $0.1 \mu \mathrm{Ci}$ of $\left[{ }^{14} \mathrm{C}\right]$ 
acetyl-CoA (40 to $60 \mathrm{mCi} / \mathrm{mmol}$; Hartmann Analytics) for $1 \mathrm{~h}$ at $30^{\circ} \mathrm{C}$. The reactions were stopped by adding $4 \times$ Laemmli buffer and separated on a 4-to- $12 \%$ polyacrylamide gel. Gels were fixed in fixation solution $(5 \%$ [vol/vol] methanol and $10 \%$ [vol/vol] acetic acid) for $30 \mathrm{~min}$. Gels were then dried and placed in a phosphorimager cassette for 10 days at room temperature. The SDS-PAGE gel was run in duplicate and stained with Coomassie blue to visualize proteins.

\section{Mass spectrometric analysis of phosphorylation sites.}

MALDI-TOF MS/MS analyses were carried out on MBPtagged NbREM4 in the presence of NbPBS1 and kinase buffer with nonradioactive ATP. The reaction was incubated for $1 \mathrm{~h}$ at room temperature, stopped by adding $4 \times$ Laemmli buffer, and separated on a 4-to-12\% polyacrylamide gel. Bands corresponding to the size of MBP-NbREM4 were excised from the gel and subjected to tryptic digestion, as described earlier (Witzel et al. 2017). Phosphorylated peptides were enriched using the High-Select Fe-NTA Phosphopeptide Enrichment Kit (ThermoFisher Scientific) following the manufacturer's instructions. The resulting eluate was desalted using $\mathrm{C}_{18}$ ZipTip Pipette Tips (Merck Millipore). After mixing 1:1 with 2,5-dihydroxybenzoic acid (Bruker Daltonik $\mathrm{GmbH})\left(20 \mathrm{mg} \mathrm{ml}^{-1}\right.$ in 30:70 [ $\left.\mathrm{vol} / \mathrm{vol}\right]$ acetonitrile- $0.1 \%$ trifluoroacetic acid in water, supplemented with $1 \% \mathrm{H}_{3} \mathrm{PO}_{4}$ ) as a matrix, the sample was spotted onto a ground steel target and allowed to dry at room temperature. Mass spectrometric experiments were performed using an ultrafleXtreme MALDI-TOF instrument (Bruker Daltonik) run in positive ionization mode, controlled by flexControl v3.4 software (Bruker Daltonik). The acquired peptide mass fingerprint data were processed with flexAnalysis v3.4 (Bruker Daltonik) and matched to the amino acid sequence of MBP-tagged NbREM4 via MASCOT search engine (Matrix Science). Search parameters were monoisotopic mass accuracy, one missed cleavage, and allowed variable modification as phosphorylation. Peptide tolerance for peptide mass fingerprinting was $50 \mathrm{ppm}$ and, for de novo sequencing, peptide tolerance was $50 \mathrm{ppm}$ and $0.7-\mathrm{Da}$ fragment tolerance. Acquisition of LIFT spectra was pursued for a peptide $\mathrm{m} / \mathrm{z}, 2,474.03$, where a putative phosphorylation was detected. Peptide mass fingerprinting spectra and corresponding LIFT spectra were calibrated using external calibration (Peptide Calibration Standard II; Bruker Daltonik).

\section{ACKNOWLEDGMENTS}

We thank M. Heinze, S. Jeserigk, and K. Bieler for their skillful technical help.

\section{AUTHOR-RECOMMENDED INTERNET RESOURCE}

NetPhos3.1: http://www.cbs.dtu.dk/services/NetPhos/

\section{LITERATURE CITED}

Arsova, B., Hoja, U., Wimmelbacher, M., Greiner, E., Ustün, S., Melzer, M., Petersen, K., Lein, W., and Börnke, F. 2010. Plastidial thioredoxin $\mathrm{z}$ interacts with two fructokinase-like proteins in a thioldependent manner: Evidence for an essential role in chloroplast development in Arabidopsis and Nicotiana benthamiana. Plant Cell 22:1498-1515.

Baudin, M., Hassan, J. A., Schreiber, K. J., and Lewis, J. D. 2017. Analysis of the ZAR1 immune complex reveals determinants for immunity and molecular interactions. Plant Physiol. 174:2038-2053.

Benschop, J. J., Mohammed, S., O’Flaherty, M., Heck, A. J., Slijper, M., and Menke, F. L. 2007. Quantitative phosphoproteomics of early elicitor signaling in Arabidopsis. Mol. Cell. Proteomics 6: 1198-1214

Bi, G., Zhou, Z., Wang, W., Li, L., Rao, S., Wu, Y., Zhang, X., Menke, F. L. H., Chen, S., and Zhou, J.-M. 2018. Receptor-like cytoplasmic kinases directly link diverse pattern recognition receptors to the activation of mitogen-activated protein kinase cascades in Arabidopsis. Plant Cell 30:1543-1561.

Börnke, F. 2005. The variable C-terminus of 14-3-3 proteins mediates isoform-specific interaction with sucrose-phosphate synthase in the yeast two-hybrid system. J. Plant Physiol. 162:161-168.

Bozkurt, T. O., Richardson, A., Dagdas, Y. F., Mongrand, S., Kamoun, S., and Raffaele, S. 2014. The plant membrane-associated REMORIN1.3 accumulates in discrete perihaustorial domains and enhances susceptibility to Phytophthora infestans. Plant Physiol. 165:1005-1018.

Büttner, D. 2016. Behind the lines-actions of bacterial type III effector proteins in plant cells. FEMS Microbiol. Rev. 40:894-937.

Cesari, S. 2018. Multiple strategies for pathogen perception by plant immune receptors. New Phytol. 219:17-24.

Demir, F., Horntrich, C., Blachutzik, J. O., Scherzer, S., Reinders, Y., Kierszniowska, S., Schulze, W. X., Harms, G. S., Hedrich, R., Geiger, D., and Kreuzer, I. 2013. Arabidopsis nanodomain-delimited ABA signaling pathway regulates the anion channel SLAH3. Proc. Natl. Acad. Sci. U.S.A. 110:8296-8301.

Dodds, P. N., and Rathjen, J. P. 2010. Plant immunity: Towards an integrated view of plant-pathogen interactions. Nat. Rev. Genet. 11: 539-548.

$\mathrm{Fu}, \mathrm{S} ., \mathrm{Xu}, \mathrm{Y} ., \mathrm{Li}, \mathrm{C} ., \mathrm{Li}, \mathrm{Y} ., \mathrm{Wu}, \mathrm{J}$. , and Zhou, X. 2018. Rice stripe virus interferes with S-acylation of remorin and induces its autophagic degradation to facilitate virus infection. Mol. Plant 11:269-287.

Goodin, M. M., Zaitlin, D., Naidu, R. A., and Lommel, S. A. 2008 Nicotiana benthamiana: Its history and future as a model for plant-pathogen interactions. Mol. Plant-Microbe Interact. 21: 1015-1026.

Gronnier, J., Crowet, J.-M., Habenstein, B., Nasir, M. N., Bayle, V., Hosy, E., Platre, M. P., Gouguet, P., Raffaele, S., Martinez, D., Grelard, A. Loquet, A., Simon-Plas, F., Gerbeau-Pissot, P., Der, C., Bayer, E. M., Jaillais, Y., Deleu, M., Germain, V., Lins, L., and Mongrand, S. 2017. Structural basis for plant plasma membrane protein dynamics and organization into functional nanodomains. eLife 6:e26404.

Hicks, S. W., and Galán, J. E. 2013. Exploitation of eukaryotic subcellular targeting mechanisms by bacterial effectors. Nat. Rev. Microbiol. 11: 316-326.

Hoefle, C., and Hückelhoven, R. 2008. Enemy at the gates: Traffic at the plant cell pathogen interface. Cell. Microbiol. 10:2400-2407.

Hurley, B., Lee, D., Mott, A., Wilton, M., Liu, J., Liu, Y. C., Angers, S., Coaker, G., Guttman, D. S., and Desveaux, D. 2014. The Pseudomonas syringae type III effector HopF2 suppresses Arabidopsis stomatal immunity. PLoS One 9:e114921.

Jarsch, I. K., Konrad, S. S., Stratil, T. F., Urbanus, S. L., Szymanski, W., Braun, P., Braun, K. H., and Ott, T. 2014. Plasma membranes are subcompartmentalized into a plethora of coexisting and diverse microdomains in Arabidopsis and Nicotiana benthamiana. Plant Cell 26: 1698-1711.

Jarsch, I. K., and Ott, T. 2011. Perspectives on remorin proteins, membrane rafts, and their role during plant-microbe interactions. Mol. PlantMicrobe Interact. 24:7-12.

Jiang, S., Yao, J., Ma, K. W., Zhou, H., Song, J., He, S. Y., and Ma, W. 2013. Bacterial effector activates jasmonate signaling by directly targeting JAZ transcriptional repressors. PLoS Pathog. 9:e1003715.

Jones, J. D., and Dangl, J. L. 2006. The plant immune system. Nature 444: 323-329.

Karimi, M., Inzé, D., and Depicker, A. 2002. GATEWAY vectors for Agrobacterium-mediated plant transformation. Trends Plant Sci. 7: 193-195.

Khan, M., Seto, D., Subramaniam, R., and Desveaux, D. 2018a. Oh, the places they'll go! A survey of phytopathogen effectors and their host targets. Plant J. 93:651-663.

Khan, M., Youn, J. Y., Gingras, A. C., Subramaniam, R., and Desveaux, D. 2018b. In planta proximity dependent biotin identification (BioID). Sci. Rep. 8: Article 9212.

Keinath, N. F., Kierszniowska, S., Lorek, J., Bourdais, G., Kessler, S. A., Shimosato-Asano, H., Grossniklaus, U., Schulze, W. X., Robatzek, S., and Panstruga, R. 2010. PAMP (pathogen-associated molecular pattern)-induced changes in plasma membrane compartmentalization reveal novel components of plant immunity. J. Biol. Chem. 285: 39140-39149.

Lal, N. K., Nagalakshmi, U., Hurlburt, N. K., Flores, R., Bak, A., Sone, P., Ma, X., Song, G., Walley, J., Shan, L., He, P., Casteel, C., Fisher, A. J., and Dinesh-Kumar, S. P. 2018. The receptor-like cytoplasmic kinase BIK1 localizes to the nucleus and regulates defense hormone expression during plant innate immunity. Cell Host Microbe 23:485-497.E5.

Laluk, K., Luo, H., Chai, M., Dhawan, R., Lai, Z., and Mengiste, T. 2011. Biochemical and genetic requirements for function of the immune 
response regulator BOTRYTIS-INDUCED KINASE1 in plant growth, ethylene signaling, and PAMP-triggered immunity in Arabidopsis. Plant Cell 23:2831-2849.

Lee, A. H., Hurley, B., Felsensteiner, C., Yea, C., Ckurshumova, W., Bartetzko, V., Wang, P. W., Quach, V., Lewis, J. D., Liu, Y. C., Börnke, F., Angers, S., Wilde, A., Guttman, D. S., and Desveaux, D. 2012. A bacterial acetyltransferase destroys plant microtubule networks and blocks secretion. PLoS Pathog. 8:e1002523.

Lefebvre, B., Timmers, T., Mbengue, M., Moreau, S., Hervé, C., Tóth, K., Bittencourt-Silvestre, J., Klaus, D., Deslandes, L., Godiard, L., Murray, J. D., Udvardi, M. K., Raffaele, S., Mongrand, S., Cullimore, J., Gamas, P., Niebel, A., and Ott, T. 2010. A remorin protein interacts with symbiotic receptors and regulates bacterial infection. Proc. Natl. Acad. Sci. U.S.A. 107:2343-2348.

Lewis, J. D., Abada, W., Ma, W., Guttman, D. S., and Desveaux, D. 2008 The HopZ family of Pseudomonas syringae type III effectors require myristoylation for virulence and avirulence functions in Arabidopsis thaliana. J. Bacteriol. 190:2880-2891.

Lewis, J. D., Lee, A., Ma, W., Zhou, H., Guttman, D. S., and Desveaux, D. 2011. The YopJ superfamily in plant-associated bacteria. Mol. Plant Pathol. 12:928-937

Lewis, J. D., Lee, A. H., Hassan, J. A., Wan, J., Hurley, B., Jhingree, J. R., Wang, P. W., Lo, T., Youn, J. Y., Guttman, D. S., and Desveaux, D. 2013. The Arabidopsis ZED1 pseudokinase is required for ZAR1-mediated immunity induced by the Pseudomonas syringae type III effector HopZ1a. Proc. Natl. Acad. Sci. U.S.A. 110:18722-18727.

Lewis, J. D., Wilton, M., Mott, G. A., Lu, W., Hassan, J. A., Guttman, D. S., and Desveaux, D. 2014. Immunomodulation by the Pseudomonas syringae HopZ type III effector family in Arabidopsis. PLoS One 9: e116152.

Lewis, J. D., Wu, R., Guttman, D. S., and Desveaux, D. 2010. Allelespecific virulence attenuation of the Pseudomonas syringae HopZ1a type III effector via the Arabidopsis ZAR1 resistance protein. PLoS Genet. 6:e1000894.

Liang, P., Stratil, T. F., Popp, C., Marín, M., Folgmann, J., Mysore, K. S., Wen, J., and Ott, T. 2018. Symbiotic root infections in Medicago truncatula require remorin-mediated receptor stabilization in membrane nanodomains. Proc. Natl. Acad. Sci. U.S.A. 115:5289-5294.

Lin, W., Ma, X., Shan, L., and He, P. 2013. Big roles of small kinases: The complex functions of receptor-like cytoplasmic kinases in plant immunity and development. J. Integr. Plant Biol. 55:1188-1197.

Liu, Y., Schiff, M., and Dinesh-Kumar, S. P. 2002a. Virus-induced gene silencing in tomato. Plant J. 31:777-786.

Liu, Y., Schiff, M., Marathe, R., and Dinesh-Kumar, S. P. 2002b. Tobacco Rarl, EDS1 and NPR1/NIM1 like genes are required for $N$-mediated resistance to tobacco mosaic virus. Plant J. 30:415-429.

Lu, D., Wu, S., He, P., and Shan, L. 2010. Phosphorylation of receptor-like cytoplasmic kinases by bacterial flagellin. Plant Signal. Behav. 5: 598-600.

Ma, W., Dong, F. F., Stavrinides, J., and Guttman, D. S. 2006. Type III effector diversification via both pathoadaptation and horizontal transfer in response to a coevolutionary arms race. PLoS Genet. 2:e209.

Macho, A. P., and Zipfel, C. 2015. Targeting of plant pattern recognition receptor-triggered immunity by bacterial type-III secretion system effectors. Curr. Opin. Microbiol. 23:14-22.

Marín, M., and Ott, T. 2012. Phosphorylation of intrinsically disordered regions in remorin proteins. Front. Plant Sci. 3:86.

Marín, M., Thallmair, V., and Ott, T. 2012. The intrinsically disordered Nterminal region of AtREM1.3 remorin protein mediates protein-protein interactions. J. Biol. Chem. 287:39982-39991.

Morel, J. B., and Dangl, J. L. 1997. The hypersensitive response and the induction of cell death in plants. Cell Death Differ. 4:671-683.

Nguyen, H. P., Chakravarthy, S., Velásquez, A. C., McLane, H. L., Zeng, L., Nakayashiki, H., Park, D. H., Collmer, A., and Martin, G. B. 2010. Methods to study PAMP-triggered immunity using tomato and Nicotiana benthamiana. Mol. Plant-Microbe Interact. 23:991-999.

Ott, T. 2017. Membrane nanodomains and microdomains in plant-microbe interactions. Curr. Opin. Plant Biol. 40:82-88.

Perraki, A., Binaghi, M., Mecchia, M. A., Gronnier, J., German-Retana, S., Mongrand, S., Bayer, E., Zelada, A. M., and Germain, V. 2014. StRemorin1.3 hampers Potato virus $X$ TGBp1 ability to increase plasmodesmata permeability, but does not interfere with its silencing suppressor activity. FEBS Lett. 588:1699-1705.
Raffaele, S., Bayer, E., Lafarge, D., Cluzet, S., German Retana, S. Boubekeur, T., Leborgne-Castel, N., Carde, J.-P., Lherminier, J., Noirot, E., Satiat-Jeunemaître, B., Laroche-Traineau, J., Moreau, P., Ott, T., Maule, A. J., Reymond, P., Simon-Plas, F., Farmer, E. E., Bessoule, J.-J., and Mongrand, S. 2009. Remorin, a solanaceae protein resident in membrane rafts and plasmodesmata, impairs Potato virus $X$ movement. Plant Cell 21:1541-1555.

Raffaele, S., Mongrand, S., Gamas, P., Niebel, A., and Ott, T. 2007. Genome-wide annotation of remorins, a plant-specific protein family: Evolutionary and functional perspectives. Plant Physiol. 145:593-600.

Rao, S., Zhou, Z., Miao, P., Bi, G., Hu, M., Wu, Y., Feng, F., Zhang, X., and Zhou, J.-M. 2018. Roles of receptor-like cytoplasmic kinase VII members in pattern-triggered immune signaling. Plant Physiol. 177: 1679-1690.

Reymond, P., Kunz, B., Paul-Pletzer, K., Grimm, R., Eckerskorn, C., and Farmer, E. E. 1996. Cloning of a cDNA encoding a plasma membraneassociated, uronide binding phosphoprotein with physical properties similar to viral movement proteins. Plant Cell 8:2265-2276.

Schultink, A., Qi, T., Bally, J., and Staskawicz, B. 2019. Using forward genetics in Nicotiana benthamiana to uncover the immune signaling pathway mediating recognition of the Xanthomonas perforans effector XopJ4. New Phytol. 221:1001-1009.

Seto, D., Koulena, N., Lo, T., Menna, A., Guttman, D. S., and Desveaux, D. 2017. Expanded type III effector recognition by the ZAR1 NLR protein using ZED1-related kinases. Nat. Plants 3: Article 17027.

Shao, F., Golstein, C., Ade, J., Stoutemyer, M., Dixon, J. E., and Innes, R. W. 2003. Cleavage of Arabidopsis PBS1 by a bacterial type III effector. Science 301:1230-1233.

Son, S., Oh, C. J., and An, C. S. 2014. Arabidopsis thaliana remorins interact with SnRK1 and play a role in susceptibility to Beet curly top virus and Beet severe curly top virus. Plant Pathol. J. 30:269-278.

Stall, R. E., Bartz, J. A., and Cook, A. A. 1974. Decreased hypersensitivity to xanthomonads in pepper after inoculations with virulent cells of Xanthomonas vesictoria. Phytopathology 64:731-735.

Sun, J., Huang, G., Fan, F., Wang, S., Zhang, Y., Han, Y., Zou, Y., and Lu, D. 2017. Comparative study of Arabidopsis PBS1 and a wheat PBS1 homolog helps understand the mechanism of PBS1 functioning in innate immunity. Sci. Rep. 7: Article 5487.

Tóth, K., Stratil, T. F., Madsen, E. B., Ye, J., Popp, C., Antolín-Llovera, M., Grossmann, C., Jensen, O. N., Schüssler, A., Parniske, M., and Ott, T. 2012. Functional domain analysis of the remorin protein LjSYMREM1 in Lotus japonicus. PLoS One 7:e30817.

Wang, G., Roux, B., Feng, F., Guy, E., Li, L., Li, N., Zhang, X., Lautier, M., Jardinaud, M. F., Chabannes, M., Arlat, M., Chen, S., He, C., Noël, L. D. and Zhou, J. M. 2015. The decoy substrate of a pathogen effector and a pseudokinase specify pathogen-induced modified-self recognition and immunity in plants. Cell Host Microbe 18:285-295.

Witzel, K., Buhtz, A., and Grosch, R. 2017. Temporal impact of the vascular wilt pathogen Verticillium dahliae on tomato root proteome. J. Proteomics 169:215-224.

Yamada, K., Yamaguchi, K., Shirakawa, T., Nakagami, H., Mine, A., Ishikawa, K., Fujiwara, M., Narusaka, M., Narusaka, Y., Ichimura, K., Kobayashi, Y., Matsui, H., Nomura, Y., Nomoto, M., Tada, Y., Fukao, Y., Fukamizo, T., Tsuda, K., Shirasu, K., Shibuya, N., and Kawasaki, T. 2016. The Arabidopsis CERK1-associated kinase PBL27 connects chitin perception to MAPK activation. EMBO J. 35:2468-2483.

Zhang, J., Li, W., Xiang, T., Liu, Z., Laluk, K., Ding, X., Zou, Y., Gao, M., Zhang, X., Chen, S., Mengiste, T., Zhang, Y., and Zhou, J. M. 2010 Receptor-like cytoplasmic kinases integrate signaling from multiple plant immune receptors and are targeted by a Pseudomonas syringae effector. Cell Host Microbe 7:290-301.

Zheng, X. Y., Spivey, N. W., Zeng, W., Liu, P. P., Fu, Z. Q., Klessig, D. F., He, S. Y., and Dong, X. 2012. Coronatine promotes Pseudomonas syringae virulence in plants by activating a signaling cascade that inhibits salicylic acid accumulation. Cell Host Microbe 11:587-596.

Zhou, H., Lin, J., Johnson, A., Morgan, R. L., Zhong, W., and Ma, W. 2011. Pseudomonas syringae type III effector HopZ1 targets a host enzyme to suppress isoflavone biosynthesis and promote infection in soybean. Cell Host Microbe 9:177-186.

Zhou, H., Morgan, R. L., Guttman, D. S., and Ma, W. 2009. Allelic variants of the Pseudomonas syringae type III effector HopZ1 are differentially recognized by plant resistance systems. Mol. Plant-Microbe Interact. 22: 176-189. 